\title{
Level Curvature Distribution and the Structure of Eigenfunctions in Disordered Systems.
}

\author{
C. Basu ${ }^{1}$, C.M.Canali2团, V. E. Kravtsov ${ }^{1,4}$, I. V. Yurkevich ${ }^{5}$ \\ ${ }^{1}$ International Center for Theoretical Physics, \\ P.O.Box 586, 34100 Trieste, Italy. \\ 2 Dept. of Applied Physics, Chalmers University of Technology \\ and Göteborg University, S-412 96 Göteborg, Sweden. \\ ${ }^{4}$ Landau Institute for Theoretical Physics, \\ Kosygina str. 2, 117940 Moscow, Russia. \\ ${ }^{5}$ The University of Birmingham, School of Physics and Astronomy, \\ Edgbaston, Birmingham B15 2TT, UK. \\ (today)
}

\begin{abstract}
The level curvature distribution function is studied both analytically and numerically for the case of T-breaking perturbations over the orthogonal ensemble. The leading correction to the shape of the curvature distribution beyond the random matrix theory is calculated using the nonlinear supersymmetric sigma-model and compared to numerical simulations on the Anderson model.

It is predicted analytically and confirmed numerically that the sign of the correction is different for T-breaking perturbations caused by a constant vector-potential equivalent to a phase twist in the boundary conditions, and those caused by a random magnetic field.

In the former case it is shown using a nonperturbative approach that quasi-localized states in weakly disordered systems can cause the curvature distribution to be nonanalytic. In $2 d$ systems the distribution function $P(K)$ has a branching point at $K=0$ that is related to the multifractality of the wave functions and thus should be a generic feature of all critical eigenstates. A relationship between the branching power and the multifractality exponent $d_{2}$ is suggested. Evidence of the branch cut singularity is found in numerical simulations in $2 d$ systems and at the Anderson transition point in $3 d$ systems.
\end{abstract}

\section{INTRODUCTION.}

As first suggested by Edwards and Thouless 1 , the sensitivity of the spectrum $\left\{E_{n}\right\}$ of disordered conductors to a small twist of phase $\phi$ in the boundary conditions $\Psi(x=$ $0, \rho)=e^{i \phi} \Psi(x=L, \rho)$ is a powerful tool to probe the space structure of eigenfunctions and distinguish between the extended and the localized states. More precisely, the quantity $K_{n}$ that is now referred to as the "level curvature", was introduced in Ref. [ 1] in order to describe this sensitivity quantitatively: 


$$
K_{n}=\left.\frac{1}{\Delta} \frac{\partial^{2} E_{n}(\phi)}{\partial \phi^{2}}\right|_{\phi=0}
$$

where the mean level spacing $\Delta=\left(\nu L^{d}\right)^{-1}$ is related to the mean density of states $\nu=\langle\nu(E)\rangle$ and the size of the $d$-dimensional sample $L$.

In complex or disordered quantum systems, the quantity $K_{n}$ fluctuates over the ensemble of energy levels $\left\{E_{n}\right\}$ or, for a given level, over the ensemble of realizations of disorder. The typical width of the distribution of level curvatures $P(K)$ is of the order of the dimensionless conductance $g=D /\left(L^{2} \Delta\right)$, where $D$ is the diffusion coefficient. Thus studying this distribution one can study the Anderson transition from metal to insulator that takes place upon increasing the disorder.

Recent work $\mathrm{Q}$ has shown that the distribution of the fluctuating quantity $K_{n}$ is a particular example of parametric level statistics, i.e. statistics of spectral responses of the system to a perturbation proportional to some parameter $\phi$.

A remarkable property that the parametric level statistics 2 share with the usual level statistics B $^{\text {A }}$, is that in a certain limit they are universal for all classically chaotic and disordered systems and can be described by the random matrix theory (RMT) of Wigner and Dyson 3 . For disordered systems considered here this limit coincides 5 with $g \rightarrow \infty$. For chaotic systems the same role is played by the ratio $g=\gamma_{1} / \Delta$, where $\gamma_{1}$ is the first nonzero mode in the spectrum of the Perron-Frobenius operator that describes the chaotic behavior of the corresponding classical system. In particular, for a time-reversal-invariant system without spin-dependent interactions (orthogonal ensemble) the distribution of level curvatures, Eq.(1.1), was found 9 in this limit to have the form:

$$
P_{W D}(k)=\frac{1}{2\left(1+k^{2}\right)^{\frac{3}{2}}}, \quad k=\frac{K}{\langle|K|\rangle},
$$

where $\langle|K|\rangle_{R M T}=2 g$ is the average modulus of the level curvature. Further study 9 has shown that the the form of the curvature distribution is still given by Eq.(1.2) even when weak localization is taken into account; only the dimensionless conductance in the expression for $\langle|K|\rangle$ is changed appropriately.

The form of Eq.(1.2) is universal. It does not depend, e.g. on the details of the system and the perturbation. Its validity only requires the system to be T-invariant with $T^{2}=1$, and the perturbation to break this invariance. The underlying physics behind this universality is the basis invariance of RMT which is equivalent to the eigenfunctions of the physical system being structureless.

Anderson localization apparently breaks the basis invariance. Thus at a sufficiently small value of $g$ the universality of the spectral statistics should break down as well. In the strong localization limit one would expect $\$ 0$ a logarithmically normal decay rather than the power-law tails of the Edwards-Thouless curvature distribution function. This is because the fluctuations of level curvature $K \propto e^{-L / \xi}$ can be viewed in this case as the consequence of the Gaussian fluctuations of the localization radius $\xi$ for the exponentially localized wave functions. This picture is qualitatively confirmed by recent analytical11 and numerical 1210 calculations.

In the present paper we present a comprehensive review of our recent analytical and numerical results on the correction to the level curvature distribution $\delta P(k)=P(k)-$ 
$P_{W D}(k)$ when one approaches the Anderson transition point $g=g_{d}^{*}$ from the metal side $g \gg 1$. Some of the results discussed below are published in $19,44,13$.

It turns out that there are two completely different contributions to the correction $\delta P(k)$. One of them $\delta P_{\text {reg }}(k)$ is regular in the small parameter $g^{-1}$ and can be obtained by a perturbative treatment 15 of the nonzero spatial modes of the nonlinear supersymmetric sigma-model 1 . The main result of this treatment 14 is that the sign of the correction $\delta P_{\text {reg }}(k)$ depends on the topological nature of perturbation. It is different for the "global" EdwardsThouless curvature, where the perturbation is represented by a global twist of the phase in the boundary conditions, 16 and for the case in which the curvature is probed by a "local" T-breaking perturbation such as magnetic impurities or random magnetic fluxes. Below we present a numerical evidence of this fact.

On top of the regular correction, there is 13 also a nonperturbative in $g^{-1}$ correction $\delta P_{s}(k)$ which is proportional to $\exp \left(-1 / g^{-1}\right)$. The latter correction is due to the so called pre-localized states 1721 , i.e. eigenstates with anomalously high peak(s) in the probability density $|\Psi(\mathbf{r})|^{2}$.

There are reasons to consider the highly irregular, multifractal critical eigenstates 22 as the result of a proliferation of such pre-localized states. This point of view is partly supported by the observation that weakly localized states in the critical dimensionality $d=2$ also exhibit a (weak) multifractality, as can be shown by means of the same methods (renormalization group 23, 17 or space inhomogeneous saddle-point approximation 18 ) that were used to discover the pre-localized states responsible for the slow current relaxation in disordered conductors2320.

This idea enables us to extend the results for $\delta P_{s}(k)$ obtained by the novel saddle-point approximation 20.13 for $2 d$ metals to the critical state at the Anderson transition in $2+\epsilon$ dimensions. Thus we can explain the branching nonanalyticity at $k=0$ found numerically in 10 for the $3 d$ critical level curvature distribution function $P_{c}(k)$. Furthermore, we suggest a relationship between the branching power and the exponent $d_{2}$ describing generic multifractal critical states22. This relationship fits well the numerical results and provides a link between the spectral statistics and statistics of wavefunctions near the Anderson transition.

The paper is organized as follows. In Section II we describe the perturbative in $g^{-1}$ approach for calculating $\delta P_{\text {reg }}(k)$. In Section III we generalize the instanton approximation of Ref. [20] for the problem of level curvature distribution and calculate the nonperturbative contribution $\delta P_{s}(k)$ for the metallic (weakly-localized) states in quasi- $1 d$ and $2 d$ systems. In Section IV we extend the results of Section III to the critical states in $2+\epsilon$ dimensions and derive the relationship between the branching nonanalyticity in $P_{c}(k)$ and the fractal dimensionality $d_{2}$. In Section $\mathrm{V}$ the results of numerical simulation on the Anderson model are presented. Some open questions are discussed in the Conclusions.

\section{PERTURBATIVE CORRECTIONS TO $P(K)$.}

\section{A. Main results.}

A general approach to calculate the $1 / g$-corrections using the nonlinear supersymmetric sigma-model 5 has been suggested in 15 and applied to the distributions of different quantities 9, 15,24. It is based on a perturbative analysis of the nonzero diffusion modes 
which are integrated out to produce $1 / g$ corrections to the zero-mode supersymmetric sigmamodel 5 . The latter must then be handled exactly.

Before going into the details of the calculations we would like to formulate the main results for $\delta P_{\text {reg }}(k)$ for the case of T-breaking perturbations over the orthogonal ensemble:

$$
\delta P_{\text {reg }}(k)=C_{d} \frac{2-11 k^{2}+2 k^{4}}{2\left(1+k^{2}\right)^{7 / 2}}, \quad k=\frac{K}{\langle|K|\rangle} \ll g,
$$

where

$$
C_{d}=\frac{1}{(\pi g)^{2}} \sum_{\mathbf{q} \neq 0} \frac{1}{\left(\mathbf{q}^{2}\right)^{2}} \times\left\{\begin{array}{ll}
\left(\frac{4}{d}-1\right), & \text { case I } \\
-1, & \text { case II }
\end{array} .\right.
$$

Here $\mathbf{q}=\left\{q_{1}, \ldots q_{d}\right\}$, where $q_{i}=2 \pi n_{i},\left(n_{i}=0, \pm 1, \pm 2 \ldots\right)$ in the case of the periodic boundary conditions (for an unperturbed system) considered in this paper.

A remarkable feature of Eqs.(2.1), (2.2) for $d<4$ is that the sign of the correction is different for global (case I) and local (case II) T-breaking perturbations. See Fig. 1.

The positive sign at small $k$ in case I reflects the tendency towards a weaker spectral response to a change in the boundary conditions with decreasing $g$. It is related to longrange correlations in the wavefunctions that result in mesoscopic fluctuations of the matrix element of perturbation. The same long-range correlations cause mesoscopic fluctuations of the diffusion coefficient.

The negative sign of the correction $\delta P_{\text {reg }}(k)$ for local perturbations [case II] is entirely due to the effect of the energy level statistics that lead to mesoscopic fluctuations of the density of states. The mesoscopic fluctuations of the matrix elements are suppressed in this case, because the effect of long-range correlations of the unperturbed wavefunctions is cut by the local nature of the perturbation.

In order to illustrate the effect of the energy level statistics on the level curvature we invoke the expression for $K_{n}$ in terms of the matrix element of the perturbation $\left|V_{n m}\right|^{2}$ and the exact eigenvalues in the absence of perturbation $E_{n}$ :

$$
K_{n}=2 \sum_{m \neq n} \frac{\left|V_{n m}\right|^{2}}{E_{n}-E_{m}} .
$$

From this expression we can clearly identify the two sources of the fluctuations in the level curvature. If the fluctuations of the matrix elements are suppressed, $\left|V_{n m}\right|^{2}$ can be replaced by a constant. Then there is only one source of fluctuations left, that is the energy level statistics. Notice that implicitly this contains the effect of the statistics of the eigenfunctions as well, and in particular, their long-range correlations. Upon decreasing $g$ the system of energy levels becomes less and less correlated. In the extreme limit of uncorrelated levels the distribution of curvatures is known $\$$ to become of the Cauchy-Lorentz form $P_{C L}(k)=1 / \pi(1+$ $k^{2}$ ). Since $P_{C L}(0)=1 / \pi$ and $P_{W D}(0)=1 / 2$ we conclude that the effect of softening the energy level correlations on the shape of the curvature distribution is such that $\delta P(0)<0$, in full agreement with the results for the case II.

The principal result of this Section is that Thouless relationship of proportionality $\langle|K|\rangle \propto g$ breaks down beyond RMT. The ratio $r(g)=\langle|K|\rangle / 2 g$ increases above its RMT value $r=1$, the correction being equal to: 


$$
\delta r(g)=\frac{\delta\langle|K|\rangle}{2 g}=\frac{1}{(\pi g)^{2}} \sum_{\mathbf{q} \neq 0} \frac{1}{\left(\mathbf{q}^{2}\right)^{2}} \times\left\{\begin{array}{cl}
\left(\frac{9}{2}-\frac{16}{d}+\frac{36}{d(d+2)}\right), & \text { case I } \\
\frac{9}{2}, & \text { case II }
\end{array} .\right.
$$

\section{B. Functional representation for $P(K)$.}

We know describe how Eqs. (2.1),(2.2) have been obtained. Let us consider a disordered mesoscopic $d$-dimensional system with a random white-noise impurity potential $V(\mathbf{r})$ perturbed by a small vector-potential $\vec{\phi} / L$. It is described by the microscopic Hamiltonian of the form:

$$
H=\frac{1}{2 m}\left(\frac{1}{i} \nabla-\frac{\vec{\phi}}{L}\right)^{2}+V(\mathbf{r})
$$

In case I we assume the sample to be closed into a ring geometry pierced by a small static magnetic flux $\Phi$. Then the effect of the perturbation is equivalent to a twist of the boundary conditions generating the phase $\phi=2 \pi \Phi / \Phi_{0}\left(\Phi_{0}=h c / e\right.$ is the flux quantum). As a vector potential we will take a constant vector of the form $\vec{\phi} / L=(\phi / L) \mathbf{n}=(\phi / L)\{1,0, . .0\}$.

In the "local" case II we consider $\vec{\phi}(\mathbf{r})$ to be a random $\delta$-correlated vector-potential:

$$
\left\langle\phi_{\alpha}(\mathbf{r}) \phi_{\beta}\left(\mathbf{r}^{\prime}\right)\right\rangle=v_{\tau} \phi^{2} \delta\left(\mathbf{r}-\mathbf{r}^{\prime}\right) \delta_{\alpha \beta}, \quad v_{\tau}=\frac{D}{2 \pi \nu v_{F}^{2}}
$$

The parameter $\phi$ is introduced in this way in order to keep $\langle|K|\rangle_{R M T}=2 g$ the same as to the "global" curvature (case I).

The curvature distribution function

$$
P(K)=\Delta\left\langle\sum_{n} \delta\left(K-K_{n}\right) \delta\left(E-E_{n}\right)\right\rangle
$$

can be expressed in terms the two-level parametric correlation function $R(\omega, \phi)$

$$
R(\omega, \phi)=\nu^{-2}\langle\nu(E+\omega, \phi) \nu(E, \phi=0)\rangle
$$

in a form similar to one derived in Ref. [25] for the distribution of level velocities:

$$
P(K)=\lim _{\phi \rightarrow 0} \frac{\phi^{2}}{2} R\left(\omega=\frac{1}{2} K \Delta \phi^{2}, \phi\right) .
$$

Indeed, using the exact expression for the fluctuating density of states $\nu(E, \phi)=$ $L^{-d} \sum_{n} \delta\left(E-E_{n}(\phi)\right)$ we have:

$$
R(\omega, \phi)=\Delta^{2} \sum_{m, n}\left\langle\delta\left(\omega+E_{n}(0)-E_{m}(\phi)\right) \delta\left(E-E_{n}(0)\right)\right\rangle .
$$

Because of the level repulsion, in the limit $\omega \rightarrow 0$ and $\phi \rightarrow 0$ only terms with $n=m$ contribute to the sum Eq.(2.10). On the other hand, with a perturbation which is odd 
under time reversal, the T-invariant level energy $E_{n}(\phi) \approx E_{n}(0)+\frac{1}{2} K_{n} \Delta \phi^{2}$ must be even 26 in $\phi$. Then choosing $\omega=\frac{1}{2} K \Delta \phi^{2}$ we immediately arrive at Eq.(2.9).

The two-level correlation function $R(\omega, \phi)$ can be represented in the form of a functional integral using the Efetov's supersymmetry approach. A straightforward application of the results of Ref. [5] and Eq.(2.9) leads to:

$$
P(K)=-\left.\frac{1}{64} \frac{\partial^{2} Z}{\partial J_{1} \partial J_{2}}\right|_{J_{1}=J_{2}=0}, \quad Z=\lim _{\phi \rightarrow 0}\left\{\phi^{2} \Re \int D Q \exp (-F[Q])\right\},
$$

where for the case I the functional $F[Q]$ takes the form:

$$
F[Q]=-\frac{\pi g}{8} \int \frac{d \mathbf{r}}{V} \operatorname{Str}\left\{(L \nabla Q+i[\widehat{\phi}, Q])^{2}+2 i k \phi^{2}(\Lambda Q)\right\}+\int \frac{d \mathbf{r}}{V} \operatorname{Str}(J Q),
$$

A similar representation for $P(K)$ has been used in Ref. [9].

In Eq.(2.12) we have introduced the notation 27

$$
\begin{gathered}
\mathrm{k}=\mathrm{K} /(2 \mathrm{~g}), \\
\widehat{\phi}=\vec{\phi} P_{+} \tau, \quad J=\widehat{k}\left[J_{1} P_{+}+J_{2} P_{-}\right],
\end{gathered}
$$

and

$$
P_{+}=\frac{1}{2}(1+\Lambda), \quad P_{-}=\frac{1}{2}(1-\Lambda)
$$

The coordinate dependent $8 \times 8$ supermatrices $Q(\mathbf{r})$ are parametrized as $Q=T^{-1} \Lambda T$, where $T$ belongs to a graded coset space $\operatorname{UOSP}(2,2 \mid 4) /$

$U O S P(2 \mid 2) \otimes U O S P(2 \mid 2) 29$.

Other matrices are specified as follows:

$$
\begin{array}{ll}
\Lambda=\operatorname{diag}\left(I_{2}, I_{2},-I_{2},-I_{2}\right)_{R-A}, & \tau=\operatorname{diag}\left(\tau_{3}, \tau_{3}, 0,0\right)_{R-A}, \\
\widehat{k}=\operatorname{diag}\left(I_{2},-I_{2}, I_{2},-I_{2}\right)_{R-A}, & \tau_{3}=\operatorname{diag}(1,-1), \quad I_{2}=\operatorname{diag}(1,1) .
\end{array}
$$

Above we imply the following hierarchy of blocks of supermatrices: retarded-advanced $(R-A)$ blocks, boson-fermion $(B-F)$ blocks, and blocks corresponding to time reversal.

In the case II the linear in $\phi$ term in Eq.(2.12) is absent but otherwise the functional $F[Q]$ is the same provided that $\phi$ is introduced as in Eq.(2.6). A similar functional $F[Q]$ appears if one considers a small concentration of magnetic impurities as a perturbation. In both cases the structure of the "covariant derivative" $\mathcal{D} Q=\nabla Q+\frac{i}{L}[\widehat{\phi}, Q]$, which implies a sort of global gauge invariance, is broken down.

It is important in deriving the functional $F[Q]$ for the case II that the correlation radius of the random vector-potential is much smaller than the elastic scattering length. In this case the averaging over $\vec{\phi}(\mathbf{r})$ should be done before switching to $Q$-variables that are assumed to be slowly varying in space. In the opposite limit of large correlation radius, one should average $e^{-F[Q]}$ over $\vec{\phi}(\mathbf{r})$ and arrive at a much more complicated functional. 


\section{Perturbative treatment of nonzero modes.}

The representation given in Eqs.(2.11)-(2.12), in terms of the field $Q(\mathbf{r})$, contains all the spatial diffusion modes $\gamma_{q}=\left(D / L^{2}\right) \mathbf{q}^{2}$. However, in doing the limit $\phi \rightarrow 0$ in Eq.(2.11) the main role is played by the zero mode that corresponds to $\mathbf{q}=0$. At $\phi=0$ this mode is gapless and thus it does not cost any energy no matter how large are the components of the field $Q$ in the noncompact boson-boson sector O I $_{\text {. I }}$ is the arbitrarily large amplitudes of the zero mode components of the field $Q$ that compensate the infinitesimal parameter $\phi$ in Eqs.(2.11)-(2.12) and lead to a finite result for $P(K)$. Thus the space independent zero mode $Q_{0}$ must be considered nonperturbatively.

In the limit $g \rightarrow \infty$ all the nonzero modes can be neglectede, and one arrives 9 at the RMT result, Eq.(1.2). For finite $1 / g$ the nonzero modes should be also taken into account. However, all the nonzero modes can be treated perturbatively for $g \gg 1$ leading to some corrections to the zero-mode action. In order to obtain these corrections we have to separate the zero modes from all other modes and then integrate over all the nonzero modes using a certain perturbative scheme.

Following the method suggested by Kravtsov and Mirlin 15 we decompose matrices $Q$ (r) as follows:

$$
Q(\mathbf{r})=T_{0}^{-1} \widetilde{Q}(\mathbf{r}) T_{0}, \quad \widetilde{Q}=\Lambda \frac{1+\widetilde{W} / 2}{1-\widetilde{W} / 2}
$$

where $T_{0}$ describes the zero mode and $\widetilde{W}(\mathbf{r})=\sum_{\mathbf{q} \neq 0} \widetilde{W}_{\mathbf{q}} e^{i \mathbf{q r}}$ does not contain the zero mode at all.

As has been already noticed, the main contribution to the functional integral comes from the zero mode. The zero-mode approximation, $Q=Q_{0}=T_{0}^{-1} \Lambda T_{0}$ is known to be equivalent to RMT29. To go beyond RMT we integrate perturbatively over $\widetilde{W}(\mathbf{r})$ to obtain the effective zero-mode action $F^{e f f}\left[Q_{0}\right]$ as follows:

$$
F^{e f f}\left[Q_{0}\right]=-\ln \int D \widetilde{Q} \cdot J[\widetilde{Q}] \exp \left\{-F\left[Q_{0}, \widetilde{Q}\right]\right\}
$$

where $F\left[Q_{0}, \widetilde{Q}\right]$ is obtained from $F[Q]$ by substituting the decomposition of Eq.(2.13) and $J[\tilde{Q}]$ is the Jacobian of the corresponding nonlinear transformation.

This scheme is implemented in Appendix A. As a result we have the effective action expanded up to the second order in $1 / g$ :

$$
F^{e f f} \approx F_{0}^{e f f}+F_{1}^{e f f}+F_{2}^{e f f}+F_{J}^{e f f} .
$$

The first term in Eq.(2.14) $F_{0}^{e f f}\left[Q_{0}\right]$ is nothing but the zero-mode action responsible for the $R M T$-results22:

$$
F_{0}^{e f f}=\frac{1}{2} \alpha-i k \beta-\sum_{p} J_{p} \sigma_{p},
$$

where $p= \pm$ labels the $(R-A)$ blocks and

$$
\alpha=\operatorname{STr}\left(\hat{\phi} Q_{0}\right)^{2}, \quad \beta=S \operatorname{Tr}\left(\hat{\phi}^{2} Q_{0}\right), \quad \sigma_{p}=\operatorname{STr}\left[\widehat{k} Q_{0}^{p p}\right] .
$$


The next term $F_{1}^{e f f}\left[Q_{0}\right]$ is the first order (weak localization) correction obtained by Fyodorov and Sommers $\mathrm{\theta}$ :

$$
F_{1}^{e f f}=-\frac{1}{2} \Pi_{2} \cdot \alpha
$$

where

$$
\Pi_{2 n}=\sum_{\mathbf{q}} \Pi^{n}(\mathbf{q}) \equiv \sum_{\mathbf{q}} \frac{1}{\left(\pi g \mathbf{q}^{2}\right)^{n}}
$$

The first order correction $F_{1}^{\text {eff }}$ leads to a renormalization of the coefficient in front of $\alpha$ in $F_{0}^{e f f}$ and can be absorbed in the dimensionless conductance $g$. It can be checked that the renormalized coefficient $\bar{g}$ is exactly the conductance with weak localization corrections taken into account. In what follows we shall assume $g$ to be a renormalized conductance and we shall omit $F_{1}^{e f f}$.

We now consider the higher-order term $F_{2}^{e f f}\left[Q_{0}\right]$ :

$$
\begin{aligned}
F_{2}^{e f f} & =\Pi_{4}\left\{\left(-\frac{3}{4}+\frac{4}{d}-\frac{9}{d(d+2)}\right) \alpha^{2}+\left(\frac{7}{2}-\frac{16}{d}+\frac{36}{d(d+2)}\right) \beta^{2}\right\} \\
& +\frac{1}{2} \Pi_{4}\left(k^{2} \beta^{2}+i k(1-4 / d) \alpha \beta+\alpha\right),
\end{aligned}
$$

and a higher-order source-induced contribution $\mathcal{F}_{J}\left[Q_{0}\right]$ :

$$
F_{J}^{e f f}=\Pi_{4} \cdot\left\{\left(\frac{1}{2}(1-4 / d) \alpha-i k \beta\right) \sum_{p} J_{p} \sigma_{p}-\prod_{p} J_{p} \sigma_{p}\right\} .
$$

The terms in $F^{e f f}$ containing the factors $1 / d$ and $1 / d(d+2)$ originate, after the angular integration over q, from the gradient term linear in $\vec{\phi}, \operatorname{Str}[\vec{\phi} \nabla Q Q]$, in Eq.(2.12). Such term is present only in the "global" case I. For this reason in the "local" case II all the $d$-dependent terms in $F^{\text {eff }}$ should be omitted.

Differentiating the partition function $Z$ with respect to the sources we arrive at the expression for the level curvature distribution function $P(k)$, where $k=K /(2 \bar{g})$ :

$$
\begin{gathered}
P(k)=\lim _{\phi \rightarrow 0} \frac{-\phi^{2}}{32 \pi \bar{g}} \Re \int D Q_{0} \cdot \Phi(\alpha, \beta) \cdot \exp \left[-\frac{\alpha}{2}+i k \beta\right] S \operatorname{Str} \widehat{k} Q_{0}^{11} \operatorname{Str} \widehat{k} Q_{0}^{22}, \\
\Phi(\alpha, \beta)=1-F_{2}^{e f f}+\Pi_{4}(1+2 i k \beta-(1-4 / d) \alpha) .
\end{gathered}
$$

A remarkable feature of the perturbation theory is that the function $\Phi(\alpha, \beta)$ is a polinomial in $\alpha$ and $\beta$. This property is due to the fact that long-trace vertices that appear after the perturbative integration over $\tilde{W}(\mathbf{r})$ factorize into the product of short-trace vertices $\alpha, \beta$, and $\sigma_{p}$.

Then the correction $\delta P(K)$ can be represented as a finite order differential operator acting on the RMT distribution function $P_{W D}(K)$ that corresponds to $\Phi(\alpha, \beta)=1$ in Eq. (2.21):

$$
P(k)=\left.\Phi\left(-2 \frac{\partial}{\partial a},-i \frac{\partial}{\partial k}\right) P_{a}(k)\right|_{a=1},
$$


where

$$
\begin{aligned}
P_{a}(k) & =a^{-1} P_{W D}(k / a)= \\
& =\lim _{\phi \rightarrow 0} \frac{-\phi^{2}}{32 \pi \bar{g}} \Re \int D Q \exp \left[-\frac{\alpha}{2} a+i k \beta\right] S t r \widehat{k} Q_{0}^{11} \operatorname{Str} \widehat{k} Q_{0}^{22} .
\end{aligned}
$$

Using the identities (see Eq. A16 in Appendix A) that relate the derivatives of $P_{a}(k)$ with respect to $k$ to those with respect to $a$, we can rewrite $\delta P(k)$ in the following form:

$$
\delta P(k)=\left.\Pi_{4}\left\{\left(\frac{4}{d}-1\right) \frac{\partial^{2}}{\partial a^{2}}+\left(\frac{9}{2}-\frac{16}{d}+\frac{36}{d(d+2)}\right) \frac{\partial}{\partial a}\right\} P_{a}(k)\right|_{a=1} .
$$

Note that by the definition given in Eq.(2.23), the function $P_{a}(k)$ obeys two normalization conditions:

$$
\int d k P_{a}(k)=1 ; \quad \int d k|k| P_{a}(k)=a
$$

Using the first of these conditions we immediately conclude that the cancellation of the terms proportional to $P_{a}(k)$ in Eq.(2.24) ensures the conservation of the normalization $\int d k \delta P(K)=0$.

Next we note that the terms proportional to the first derivative can be absorbed into the function $P_{a}(k): P_{a=1}(k)+\delta(\partial / \partial a) P_{a=1}(k) \approx P_{a=1+\delta}(k)$. In doing so we observe with the help of the second normalization condition in Eq.(2.25) that $\langle|k|\rangle=\langle|K|\rangle / 2 \bar{g}=1+\delta$. Thus the terms with the first derivative in Eq.(2.24) result in a shift in the average $\langle|K|\rangle$ :

$$
\delta\langle|K|\rangle=2 \bar{g}\left(\frac{9}{2}-\frac{16}{d}+\frac{36}{d(d+2)}\right) \Pi_{4} .
$$

By redefining the $k=K /\langle|K|\rangle$, where $\langle|K|\rangle$ is the average of absolute value of the level curvature, one can cancel the terms with the first derivative in Eq.(2.24). All what is left is the term with the second derivative which describes the change in the shape of the distribution function. The final result of these tedious calculations is very simple:

$$
\delta P(k)=\left.\left(\frac{4}{d}-1\right) \Pi_{4} \frac{\partial^{2}}{\partial a^{2}}\left(a^{-1} P_{W D}(k / a)\right)\right|_{a=1}, \quad \Pi_{4} \propto 1 / \bar{g}^{2} .
$$

This equation means that the RMT curvature distribution $P_{W D}(k)$ plays the role of the generating function for its own corrections.

\section{THE SIGNATURE OF THE PRE-LOCALIZED STATES IN THE LEVEL CURVATURE DISTRIBUTION.}

It has been known for quite a while that the relaxation of current and the local density of states (DOS) in disordered conductors exhibit an anomaly even in the weak-localization regime. Namely, it has been shown in Ref. [ 17] that there exists a small (but not exponentially small) probability of finding a current relaxation time or a local DOS that is much larger than the corresponding mean values. These anomalies have been attributed to 
quasi-localized (or pre-localized) statest 20, that is, states with an anomalously large peak in $|\Psi(\mathbf{r})|^{2}$ at some point $\mathbf{r}=\mathbf{r}_{0}$.

Very recently the problem of current relaxation in disordered conductors has been reconsidered 20.30 by an elegant instanton approximation 20 applied to the supersymmetric version of the nonlinear sigma-model 1 . In these papers the main result of the previous work 17 has been confirmed for $2 d$ systems. However, the new method was able to describe some unknown regimes of current relaxation and to set correct limits of validity for the regimes found earlier. Later the same idea 20 has been applied 18 to find directly the distribution of $|\Psi(\mathbf{r})|^{2}$ and the distribution of local densities of states 31 . Thus the existence of quasi-localized states has been proved and the corresponding configuration of the random impurity potential has been found 20,21.

The main idea of Edwards and Thouless 1 is that it is possible to distinguish between localized and extended states by analyzing the sensitivity of the spectrum to a twist of the phase in the boundary conditions. This sensitivity is significant only for states with a localization radius larger than the sample size $L$ and negligible for strongly localized states. It is clear that the existence of the pre-localized states should lead to an enhancement in $P(K)$ at small $K$. In low dimensional systems $d=1,2$ where the pre-localized states correspond to localized states with an anomalously small localization radius, one may expect a singularity in $P(K)$ at $K=0$. In $3 d$ metal the typical pre-localized state looks like a sharp peak in $|\Psi(\mathbf{r})|^{2}$ on top of the extended background $|\Psi(\mathbf{r})|^{2} \sim L^{-d}=$ const. The level curvature that corresponds to such a state does not vanish but only slightly decreases. Thus the prelocalized states in $3 d$ in the weak localization regime should have much weaker effect on the level curvature distribution.

\section{A. Instanton approximation.}

In order to check these predictions we consider, instead of $P(K)$ at small $K$, its Fouriertransform $\tilde{P}(\lambda)=\int d K P(K) e^{-i K \lambda}$ at $\lambda \gg 1$.

It is easy to see that for both the RMT result Eq.(1.2) and the regular correction Eq.(2.1) the function $\tilde{P}(\lambda)$ vanishes exponentially for $\lambda \gg 1$. In what follows we will seek for slowly decreasing contributions. Support for the existence of such contributions can be gained by noticing that in the functional representation of $P(K)$, Eq.(2.11), Eq.(2.12), the level curvature $k$ plays the same role as the frequency in the problem of the current relaxation in disordered conductor $\$ 20$, thus $\lambda$ being analogous to time. Therefore, one may expect long

nonexponential tails in $\tilde{P}(\lambda)$ in analogy with those present in the current relaxation function $I(t)$. However, it is far from clear that two problems are equivalent, since the boundary conditions are different and the nonlinear sigma-model in Eq.(2.12) contains additional terms that describe the T-breaking perturbation.

The main idea of Ref. [20], that we will exploit here, is that at large $\lambda$ the configurations of the field $Q(\mathbf{r})$ that are space independent or slowly varying in space, are energetically unfavorable. In contrast, essentially space-dependent configurations in the vicinity of the classical (instanton) solution $Q_{\text {ins }}(\mathbf{r})$ that minimizes the action $F[Q]$ appear to be energetically advantageous. At large $g$ the fluctuations around this solution are expected to be small and one arrives at: 


$$
\tilde{P}(\lambda) \equiv A e^{-S(\lambda)}=\lim _{\phi \rightarrow 0} \Re \int \mathcal{D} Q \int_{-\infty}^{+\infty} d K A\left[Q_{i n s} ; \phi\right] e^{-\left\{F\left[Q_{i n s}\right]+i K \lambda\right\}} .
$$

where $A\left[Q_{\text {ins }} ; \phi\right]$ is a pre-exponential factor including the effect of fluctuations around the instanton solution.

The Grassmann variables in the action, Eq. 2.12) can lead only to a renormalization of the pre-exponential factor $A$ in Eq.(3.1), since the integration over these variables is equivalent to a differentiation. Thus with exponential accuracy we can neglect all the Grassmann variables in the Efetov's parametrization for $Q(\mathbf{r})$. Next, a finite contribution to $S(\lambda)$ in the limit $\phi \rightarrow 0$ comes only from the infinitely large boson-boson components of the field $Q(\mathbf{r})$. Therefore we consider only the leading terms in the noncompact angles $\theta_{1}$ and $\theta_{2}$ in the Efetov's parametrization for the orthogonal ensemble:

$$
Q=V^{-1} H V
$$

where

$$
H=\left(\begin{array}{ll}
\cosh \theta_{B} & -\sinh \theta_{B} \\
\sinh \theta_{B} & -\cosh \theta_{B}
\end{array}\right)_{R-A} \otimes P_{B}
$$

with $P_{B}=(\hat{k}+1) / 2$,

$$
\begin{aligned}
\cosh \theta_{B} & =\cosh \theta_{1} \cosh \theta_{2}+\sigma_{x} \sinh \theta_{1} \sinh \theta_{2}, \\
\sinh \theta_{B} & =\sinh \theta_{1} \cosh \theta_{2}+\sigma_{x} \cosh \theta_{1} \sinh \theta_{2},
\end{aligned}
$$

and

$$
V=e^{i \varphi \sigma_{z}} \otimes P_{+} \otimes P_{B}+e^{i \chi \sigma_{z}} \otimes P_{-} \otimes P_{B}
$$

The field $Q_{\text {ins }}(\mathbf{r})$ must obey periodic boundary conditions, since the twist of phase $\phi$ is taken into account explicitly in the action $F[Q]$. This means that $\theta_{1,2}(\mathbf{r})$ and the functions $\exp [\varphi(\mathbf{r})], \exp [\chi(\mathbf{r})]$ should obey periodic boundary conditions.

In this way we obtain $F\left[Q_{i n s}\right]=L^{-d} \int f\left[Q_{\text {ins }}\right] d^{d} \mathbf{r}$, where:

$$
\begin{aligned}
& f[Q]=\frac{\pi}{4} g\left[\left(\partial \theta_{+}\right)^{2}+\left(\partial \theta_{-}\right)^{2}\right]+ \\
+ & \frac{\pi}{2} g\left[(\partial \varphi-\phi \mathbf{n})^{2}+(\partial \chi)^{2}\right]\left(\cosh \theta_{+} \cosh \theta_{-}-1\right)- \\
- & 2 \partial \chi(\partial \varphi-\phi \mathbf{n}) \sinh \theta_{+} \sinh \theta_{-}-\frac{i \pi}{4} K \phi^{2}\left(\cosh \theta_{+}+\cosh \theta_{-}\right) .
\end{aligned}
$$

Here $\theta_{ \pm}=\left(\theta_{1} \pm \theta_{2}\right) / 2$ with $\theta_{1,2} \geq 0$ and $\partial \equiv\left\{\partial / \partial x_{\alpha}\right\}$.

We will look for a minimum of the functional $F\left[Q_{i n s}\right]+i K \lambda$ that corresponds to:

$$
\theta_{-}=\partial \chi=0, \quad \theta_{+} \equiv \theta \in[0,+\infty]
$$

By varying the functional $F\left[Q_{i n s}\right]+i K \lambda$ over $\theta, \varphi$ and $k$ we find:

$$
\partial^{2} \theta+\phi^{2}\left[\kappa-(\partial v-\mathbf{n})^{2}\right] \sinh \theta=0,
$$




$$
\partial[(\partial v-\mathbf{n})(\cosh \theta-1)]=0
$$

and

$$
\frac{\pi}{4} \phi^{2} \int(\cosh \theta+1) d^{d} \rho=\lambda
$$

where $\kappa=i K / 2 g, d^{d} \rho=\frac{d^{d} \mathbf{r}}{L^{d}}$ and $\varphi=\phi v$.

Eqs.(3.8)-(3.10) correspond to the global case I. As usual, in the local case II the terms linear in $\mathbf{n}=\{1,0,0, \ldots\}$ are absent.

The limit $\phi \rightarrow 0$ is done simply by absorbing $\phi^{2}$ into $\theta$. We introduce $\tilde{\theta}=\theta+\ln \phi^{2}$. Then in the limit $\phi \rightarrow 0$ we have $\sinh \theta \approx \cosh \theta=\frac{1}{2} e^{\tilde{\theta}} \phi^{-2}$ and Eqs.(3.8),(3.9), (3.10) take the form:

$$
\begin{gathered}
\partial^{2} \tilde{\theta}+\frac{1}{2}\left[\kappa-(\partial v-\mathbf{n})^{2}\right] e^{\tilde{\theta}}=0 \\
\partial\left[(\partial v-\mathbf{n}) e^{\tilde{\theta}}\right]=0 \\
\frac{\pi}{8} \int e^{\tilde{\theta}} d^{d} \rho=\lambda .
\end{gathered}
$$

where now $\tilde{\theta} \in[-\infty,+\infty]$.

Using Eqs.(3.6), (3.7), (3.11) and the periodicity of the function $\tilde{\theta}(\mathbf{r})$ we find:

$$
S(\lambda)=\frac{\pi}{4} g \int(\partial \tilde{\theta})^{2} d^{d} \rho+2 g \kappa \lambda .
$$

We note that $\phi$ drops from the problem only if we assume a topologically trivial solution corresponding to periodic boundary conditions being imposed on $v(\mathbf{r})$. Otherwise $\phi$ appears in the boundary condition for $v=\varphi(\mathbf{r}) / \phi$ which is periodic modulus $2 \pi / \phi$ and thus is ill defined in the limit $\phi \rightarrow 0$. In what follows we consider only such a topologically trivial solution.

One can solve Eq.(3.12):

$$
(\partial v-\mathbf{n})=[\nabla \times \mathbf{A}] e^{-\tilde{\theta}}
$$

where $[\nabla \times \mathbf{A}]=$ const in $1 d$ and is the curl of an arbitrary vector function $\mathbf{A}(\mathbf{r})$ in higher dimensions. Below we consider only the simplest solution that corresponds to $[\nabla \times \mathbf{A}] \equiv$ $-\mathbf{n} / N=$ const.

Let us consider first the local case II. Doing the space integration of Eq.(3.15) which in this case does not contain the term proportional to $\mathbf{n}$, and using periodic boundary conditions for $v(\mathbf{r})$ one immediately arrives at $[\nabla \times \mathbf{A}]=\partial v=0$. Then the same procedure with Eq.(3.11) leads to the conclusion that the only solution for $\tilde{\theta}$ that obeys periodic boundary conditions, is space-independent and exists only for $\kappa=\mathbf{n}^{2}=1$. The corresponding action is $S(\lambda)=2 g \lambda$. Thus the instanton approximation in the local case II gives only an exponentially small tail $\tilde{P}(\lambda) \propto e^{-2 g \lambda}$ that has been already obtained by the perturbative 
approach. We conclude that for case II the analogy with the problem of current relaxation appears to be wrong.

Now consider the global case I. Integrating Eq.(3.15) over space and using the periodicity of $v(\mathbf{r})$ gives:

$$
N=\int e^{-\tilde{\theta}} d^{d} \rho
$$

Substituting Eqs.(3.15),(3.16) into Eq.(3.11) we finally arrive at:

$$
\partial^{2} \tilde{\theta}+\frac{\partial U}{\partial \tilde{\theta}}=\partial^{2} \tilde{\theta}+\frac{\kappa}{2} e^{\tilde{\theta}}-\frac{1}{2 N^{2}} e^{-\tilde{\theta}}=0 .
$$

It appears that the global nature of perturbation and the corresponding linear in $\mathbf{n}$ term in Eq.(3.15) leads to a term proportional to $e^{-\tilde{\theta}}$ in Eq.(3.17) that builds a second "wall" in the effective "potential" $U(\tilde{\theta})$ and makes it possible for periodic solutions ("oscillations") to exist.

Eq.(3.17) takes a more symmetric form if we make a shift $\tilde{\theta}=u-\zeta$, where:

$$
\cosh \zeta=\left(\kappa+\frac{1}{N^{2}}\right) \frac{N}{2 \sqrt{\kappa}}, \quad \sinh \zeta=\left(\kappa-\frac{1}{N^{2}}\right) \frac{N}{2 \sqrt{\kappa}}
$$

Finally we have the system of equations:

$$
\begin{gathered}
\partial^{2} u+\gamma^{2} \sinh u=0, \\
\frac{1}{N}=\gamma^{2} \int e^{-u} d^{d} \rho, \\
\lambda=\frac{\pi}{8 \gamma^{2} N^{2}} \int e^{u} d^{d} \rho,
\end{gathered}
$$

where $\gamma^{2}=\sqrt{\kappa} / N$.

Solving these equations for a hyper-cubic sample $-1 / 2<\rho_{i}<1 / 2$ with periodic boundary conditions one finds $u(\mathbf{r}, \lambda), N(\lambda)$ and $\gamma(\lambda)$ which enter the instanton action $S(\lambda)$ :

$$
S(\lambda)=\frac{\pi}{4} g \int(\partial u)^{2} d^{d} \rho+2 g \gamma^{4} N^{2} \lambda
$$

\section{B. Non-exponential tails of $\tilde{P}(\lambda)$ in low-dimensional systems.}

We will see below that for large $\lambda$ the parameter $\gamma$ is small. For $\gamma \ll 1$ the term $\gamma^{2} \sinh u$ is very small unless $\sinh u$ is exponentially large. This means that we can approximate $\gamma^{2} \sinh u \approx \frac{\gamma^{2}}{2} e^{|u|} \operatorname{sign}(u)$. Thus Eq.(3.19) is replaced by the Liouville equation :

$$
\partial^{2} u+\frac{\gamma^{2}}{2} e^{|u|} \operatorname{sign}(u)=0
$$


The generic solution to the Liouville equation in the low-dimensional case $d=1,2$ is given in terms of two arbitrary functions $f(w)$ and $v(w)$ of the complex variable $w=i \gamma z / \sqrt{8}$ with $z=x+i y:$

$$
e^{|u|}=\frac{2 f^{\prime}(w) v^{\prime}\left(-w^{*}\right)}{\left(f(w)+v\left(-w^{*}\right)\right)^{2}}
$$

where $f^{\prime}=d f / d w$ and $v^{\prime}=d v / d w$. We need the r.h.s. of Eq.(3.24) to be real positive. This can be done by the choice:

$$
f^{*}(w) v\left(-w^{*}\right)=1
$$

Then we have the solution in terms of one function only $F(z)=f(i \gamma z)$ :

$$
e^{|u|}=\frac{16}{\gamma^{2}} \frac{\left|\frac{d F}{d z}\right|^{2}}{\left(1+|F|^{2}\right)^{2}}
$$

\section{Quasi-1d Case}

Choosing $F(z)=e^{k z+b}$ in Eq.(3.26), one has a quasi-1d solution to the Liouville equation that depends only on one coordinate $x$ :

$$
e^{|u|}=\frac{4 k^{2}}{\gamma^{2} \cosh ^{2}(k x+b)},
$$

where $k$ and $b$ are real constants.

The solution on a ring $-\frac{1}{2}<x<\frac{1}{2}$ is constructed by reflecting anti-symmetrically the positive solution with $b=0$ around the points $x= \pm \frac{1}{4}$ [see Fig. 2]. The second constant $k$ is found from the condition $u( \pm 1 / 4)=0$ :

$$
4 k^{2}=\gamma^{2} \cosh ^{2}(k / 4), \quad k \approx \ln \left(1 / \gamma^{4}\right)
$$

The anti-symmetry of the solution immediately leads to the identity:

$$
I=\int e^{u} d x=\int e^{-u} d x
$$

Since the function $u \sim \ln \left(1 / \gamma^{2}\right)$ is large everywhere except in the vicinity of its zeros at $|x|=\frac{1}{4}$ we have:

$$
I \approx \int_{-\frac{1}{4}}^{+\frac{1}{4}} e^{u} d x=\frac{8 k}{\gamma^{2}} \tanh (k / 4) \approx \frac{8 k}{\gamma^{2}} .
$$

Next we calculate the integral:

$$
\int_{-\frac{1}{2}}^{+\frac{1}{2}}\left(u^{\prime}\right)^{2} d x=2 \int_{-\frac{1}{4}}^{+\frac{1}{4}}\left(u^{\prime}\right)^{2} d x=4 k^{2}-16 k \approx 4 k^{2} .
$$


Then from Eqs.(3.20),(3.21) we have:

$$
\begin{gathered}
N=\frac{1}{8 k}, \\
\gamma^{4}=\frac{64 \pi k^{3}}{\lambda} .
\end{gathered}
$$

Finally using Eq.(3.22),(3.28) and (3.33) we arrive at:

$$
S(\lambda)=\pi g k^{2} \approx \pi g \ln ^{2} \lambda .
$$

Thus the characteristic function in a quasi-1d systems is:

$$
\tilde{P}(\lambda)=A \exp \left[-\frac{g_{1}}{2} \ln ^{2} \lambda\right], \quad g_{1}=2 \pi g,
$$

The above result holds within the domain of validity of the nonlinear sigma-model Eq.(2.12). This model and hence the saddle-point equations work only for sufficiently slow varying fields $Q(\mathbf{r})$, namely $|\partial u|<L / l$, where $l$ is the elastic scattering length. It follows immediately from Eqs.(3.27), (3.28) that the above result is valid for $1 \ll \lambda \ll \exp (L / l)$.

The logarithmically-normal tail in $\tilde{P}(\lambda)$ described by Eq. 3.35) is exactly of the same functional form as the current relaxation function $I(t)$ in Ref. [20] for the orthogonal ensemble.

\section{2. $2 d$ Case}

In full analogy with the quasi- $1 d$ case, we construct a double-periodic solution to the Liouville equation on a torus $-\frac{1}{2}<x, y<\frac{1}{2}$ by reflection. We consider a positive solution $u(z)$ inside the square $\Omega$ with vertices at $z= \pm 1 / 2, \pm i / 2$ and then continue it anti-symmetrically about a side of the square in any quarter of the sample $|\Re z|<1 / 2,|\Im z|<1 / 2$. The definition domain of the solution with its sign is drawn in Fig. 3. By construction, the symmetry relationship Eq.(3.29) is valid for such a $2 d$ solution too.

The procedure of finding the solution is described in the Appendix Q. We note that for our purposes we need only the solution for $|z|=r \ll 1$. It is rotationally invariant and has the form:

$$
e^{u(r)}=\frac{16 b(k-1)^{2} r^{2 k-4}}{\left(\gamma^{2}+b r^{2 k-2}\right)^{2}}
$$

where

$$
b=16\left(\frac{\pi^{2}}{2}\right)^{k}(k-1)^{2} .
$$

Note that the solution Eq.(3.36) can be immediately obtained from the radial Liouville equation, with $k$ and $b$ being two constants of integration. The requirement of periodicity of $u(z)$ helps to establish a connection, Eq.(3.37), between these constants. 
The remaining constant $k$ is found in a standard way from the requirement of convergence of $\int(\partial u)^{2} d x d y$ in the action $S(\lambda)$ :

$$
(\partial u)^{2}=\left[\frac{2 k-4}{r}-4 b(k-1) \frac{r^{2 k-3}}{\gamma^{2}+b r^{2 k-2}}\right]^{2} .
$$

Thus we immediately find:

$$
k=2, \quad b=4 \pi^{4}
$$

and

$$
(\partial u)^{2}=\frac{16 b^{2} r^{2}}{\left(\gamma^{2}+b r^{2}\right)^{2}} .
$$

Because of the symmetry of $u(z)$, the integral $\int_{\Omega}(\partial u)^{2} d^{2} \rho$ over the square $\Omega$ is exactly one-half of the total integral over the period (over the sample) $\int(\partial u)^{2} d^{2} \rho$. It diverges logarithmically at $r \gg \gamma$, and we arrive at:

$$
\int(\partial u)^{2} d^{2} \rho=32 \pi \ln \left(\frac{C b}{\gamma^{2}}\right),
$$

where $C=\frac{2}{\pi^{2} e}$ can be found from an exact solution in the region $|z| \sim 1$.

The result is almost independent of $b$ at small $\gamma$ and is essentially determined by the logarithmic solution of the Poisson equation that follows from Eq.(3.19) at $\gamma=0$.

Now let us calculate the integrals in the self-consistency equations. For symmetry reasons we have:

$$
\int e^{u} d x d y=\int e^{-u} d x d y=\frac{16 \pi(k-1)}{\gamma^{2}}=\frac{16 \pi}{\gamma^{2}}
$$

Most of the contribution to these integrals comes form the small $r \sim \gamma \ll 1$ and the result is independent of $b$.

Now we are in the position to calculate the constants $\gamma$ and $N$ that enter the instanton action, Eq.(3.22). They are given by Eqs.(3.20), (3.21),(3.42):

$$
N=\frac{1}{16 \pi}, \quad \gamma^{4}=\frac{8^{3} \pi^{4}}{\lambda}
$$

Then the final expression for the instanton action in $2 d$ reads:

$$
S(\lambda)=4 \pi^{2} g\left[\ln \left(\frac{\lambda}{8}\right)-1\right] .
$$

Accordingly, the characteristic function $\tilde{P}(\lambda)$ turns out to have a power-law asymptotic behavior at large $\lambda \gg 1$ :

$$
\tilde{P}(\lambda)=A\left(\frac{c}{\lambda}\right)^{2 g_{2}}, \quad g_{2}=2 \pi^{2} g
$$

where $c=8 e$.

Few notes should be made on the validity of the result Eq.(3.45). Firstly, the above instanton approximation with the action $S(\lambda)$ logarithmic in $\lambda$ is only justified when $g \gg$ 1, since the pre-exponential factor $A$ could also be a power-law function of $\lambda$ but with an exponent of order 1. Secondly, the nonlinear sigma-model and hence the saddle-point equations work only for $|\partial u|<L / l$, where $l$ is the elastic scattering length. It follows immediately from Eq.(3.40), (3.43) that the above result is valid for $1 \ll \lambda \ll(L / l)^{4}$. 


\section{Nonanalyticity of the level curvature distribution.}

In this section we show that the slowly decreasing tails in the characteristic function $\tilde{P}(\lambda)$ at $\lambda \gg 1$ given by Eqs.(3.35), (3.45) result in a nonanalytic behavior of $P(K)$ at $K=0$. As usual, true nonanalyticity arises only in the thermodynamic limit $L / l \rightarrow \infty 32$, since only in this limit the tails extend to infinity. For any finite $L / l$ the function $P(K)$ is still analytic at $K=0$ but the region of the regular behavior of $P(K)$ shrinks to zero with increasing $L / l$. Below the limit $L / l \rightarrow \infty$ is assumed.

Let us consider the quasi- $1 d$ case first. In this case all derivatives of $P(K)$ are finite at $K=0$ :

$$
P^{(2 n)}(0)=\int_{-\infty}^{+\infty}(-1)^{n} \lambda^{2 n} \tilde{P}(\lambda) \frac{d \lambda}{2 \pi} \propto \exp \left[(2 n+1)^{2} / 2 g_{1}\right] .
$$

Yet the function $P(K)$ is nonanalytical at $\mathrm{K}=0$, since the Taylor series $P(K)=$ $\sum_{n} \frac{P^{(2 n)}(0)}{(2 n) !} K^{2 n}$ has zero radius of convergence because of the very fast growth of $P^{(2 n)}(0)$ with $n$.

The singularity at $K=0$ is much stronger in $2 d$ case. In this case all derivatives $P^{(2 n)}(0)$ with $2 n+1>2 g_{2}$ are proportional to $(L / l)^{4\left(2 n+1-2 g_{2}\right)}$ and diverge in the thermodynamic limit. Let us define $m$ as an integer obeying the inequality of $\left|g_{2}-m\right| \leq \frac{1}{2}$. Then the the expansion of $P(K)$ at small $K$ has the form:

$$
P(K)=c_{0}+c_{1} K^{2}+\ldots c_{m-1} K^{2(m-1)}+c_{m} K^{2 m-\alpha_{m}}+o\left(K^{2 m}\right),
$$

where $0<\alpha_{m}<2$ is given by:

$$
\alpha_{n}=(2 n+1)-2 g_{2} .
$$

In the $3 d$ metal case we failed to find a solution to the saddle-point problem that would lead to a finite action $S(\lambda)$ in the thermodynamic limit. This means that the characteristic function $\tilde{P}(\lambda)$ has only regular corrections at $g \gg 1$ and thus decays exponentially for $\lambda \gg 1$.

\section{NON-ANALYTICITY OF $P(K)$ AT $K=0$ AND MULTIFRACTALITY OF EIGENFUNCTIONS.}

From the results of the previous section we see that the strength of the singularity of $P(K)$ at $K=0$ depends on the dimensionality in a nonmonotonic way. The singularity is very weak in a quasi-1d metal; it reaches a maximum in a $2 d$ metal where $P(K)$ has a branch cut; it disappears in a $3 d$ metal where the level curvature distribution is analytic.

Such a behavior is related to the fact that $d=2$ is the low critical dimension for the Anderson transition, and the wavefunctions in the $2 d$ weak-localization regime share some features of the critical wavefunctions at the Anderson transition in higher dimensions.

\section{A. $P(K)$ at the Anderson transition in $2+\epsilon$ dimensions.}

The usual way to describe the critical state near the Anderson transition is the $(d-2)=\epsilon$ expansion. To this end one considers the quantity of interest in a $2 d$ system with $g_{2} \gg 1$ 
and then replaces $g_{2}$ by the critical conductance $g_{d}^{*}=1 /(d-2)$ which is the fixed point of the scaling equation 33.34 :

$$
\frac{d \ln g_{d}}{d \ln L}=(d-2)-\frac{1}{g_{d}}+o\left(\frac{1}{g_{d}^{2}}\right) .
$$

For the orthogonal ensemble in $d=2+\epsilon$ dimensions we find to the leading order in $\epsilon \ll 1$ :

$$
\tilde{P}_{c}(\lambda) \propto\left(\frac{1}{\lambda}\right)^{\frac{2}{\epsilon}}
$$

Note that at the critical point, the conductance $g_{d}^{*}$ is exactly size-independent, and one can consider the thermodynamic limit $L \rightarrow \infty$ without tuning other parameters in order to keep $g_{d}^{*}$ fixed.

Thus one can define the critical exponent $\mu$ that determines the power-law tail of the critical characteristic function:

$$
\tilde{P}_{c}(\lambda) \propto \lambda^{-\mu}, \quad \mu=\frac{2}{\epsilon}+o(1) .
$$

If we set $\epsilon=1$ in the above equations we find $\mu \approx 2$. Then it follows from Eq.(3.47) that already the second derivative of $P(K)$ at $K=0$ is divergent:

$$
P_{c}(K)=c_{0}-c_{1}|K|^{2-\alpha}, \quad \alpha=3-\mu .
$$

\section{B. Exponent $\mu$ and multifractality.}

Unfortunately it is known that the accuracy of the $d-2=\epsilon$ expansion is quite poor and insufficient for a precise determination of the critical exponents. In this situation one can try to find relationships between different critical exponents rather than try to evaluate them using the $\epsilon$-expansion. This certainly requires some assumptions about the underlying physics.

As has been mentioned in the Introduction, a unique property of the critical states is multifractality. This property is characterized by the power-law dependences of averaged powers of eigenfunction amplitudes $\left|\Psi_{E}(\mathbf{r})\right|$. Two of such power-law dependences are known $39,22$. One of them determines the scaling of a single eigenfunction with respect to the size of the system $L$.

$$
\sum_{\mathbf{r}, n}\left\langle\left|\Psi_{n}(\mathbf{r})\right|^{2 q} \delta\left(E-E_{n}\right)\right\rangle \propto L^{-d_{q}(q-1)},
$$

Another one determines the correlations of different eigenfunctions as a function of energy difference:

$$
\sum_{\mathbf{r}, n, m}\left\langle\left|\Psi_{n}(\mathbf{r})\right|^{q}\left|\Psi_{m}(\mathbf{r})\right|^{q} \delta\left(E-E_{n}\right) \delta\left(E^{\prime}-E_{m}\right)\right\rangle \propto\left|E-E^{\prime}\right|^{-\left(1-\frac{d_{q}}{d}\right)(q-1)} .
$$

In Eqs.4.5), (4.6) $d_{q}<d$ is a fractal dimension that depends on $q$ ("multifractality"). 
It is remarkable that Eq. (4.5) can be derived 18 for the case of $2 d$ metals by means of an instanton approximation similar to the one we used in this paper. The spectrum of the fractal dimensions $d_{q}$ obtained in this approximation turns out to be linear 38 :

$$
d_{q}=d-\frac{\eta}{2} q, \quad \eta=d-d_{2}=\frac{2}{\beta g_{2}}
$$

where $\beta=1,2,4$ for the orthogonal, unitary and symplectic ensembles.

It is reasonable to assume that the power-law tail in $\tilde{P}(\lambda)$ is another signature of multifractality 10 . Then one may hope that the expression for $\mu$ in terms of the structural constant of multifractality $\eta=d-d_{2}$ provides a better approximation for $\mu$ than the $\epsilon$-expansion. By using Eq.4.7) and the relationship $39 \mu=2 \beta g_{2}$, we obtain:

$$
\mu=\frac{4}{\eta}
$$

The derivation of Eq.(4.8) that we have just carried out for the $d=2$ case is based on two crucial facts: i). the exponent $\mu$ is determined by the spectrum of multifractality $d_{q}$ and ii). this spectrum is linear (for $q \ll 1 / \eta$ ). We will now make the assumption that $\mathrm{i}$ ). is valid for any critical state. Since for any critical state with weak multifractality the spectrum of $d_{q}$ is expected to be linear up to very large values of $q$, we believe that Eq.(4.8) is valid for any critical state with weak multifractality. In contrast to Eq.(4.3), the relationship between $\mu$ and $\eta$ Eq.(4.8) is independent of dimensionality and the symmetry parameter $\beta$ and should apply to $2 d$ critical states in the Quantum Hall regime and for systems with spin-orbit interaction 35.22 .

\section{NUMERICAL EVALUATION OF $P(K)$ FOR THE ANDERSON MODEL.}

For our numerical analysis we consider a tight-binding model on a square lattice of $L^{d}$ sites. The one-particle Hamiltonian is:

$$
H=\sum_{i} \epsilon_{i} c_{i}^{\dagger} c_{i}+t \sum_{\langle<i j\rangle>}\left(e^{i \theta_{i j}} c_{i}^{\dagger} c_{j}+e^{-i \theta_{i j}} c_{j}^{\dagger} c_{i}\right)
$$

The site energies $\epsilon_{i}$ are randomly distributed with uniform probability between $-W / 2$ and $W / 2$ in units of $t=1$. The parameter $W$ controls the amount of disorder in the system. The phase shifts $\theta_{i j}$ in the hopping term connecting nearest neighbors represent the effect of an external perturbation that breaks the T-invariance of the system. As for the analytical calculations we consider two types of such perturbations. The first one (case I) is the usual Aharonov-Bohm flux $\Phi=(\phi / 2 \pi) \Phi_{0}$ that pierces the system closed to a ring geometry giving rise to a global shift of the boundary conditions in one direction. We will choose a gauge such that each hop in $x$-direction picks up a phase $\theta_{i j}=\phi / L$, so that total twist of the boundary condition is $\phi$. The second one (case II) is a random magnetic flux. In this case the gauge is such that the phase $\theta_{i j}$ relative to a hop in the $x$-direction is Gaussian distributed with zero average and variance equal to $\left\langle\theta_{i j}^{2}\right\rangle=(\phi / L)^{2}$. For this gauge, the vector-potential $\mathbf{A}(i) \propto\left\{\theta_{i, i+1}, 0, \ldots\right\}$ is defined on the dual lattice with sites in the middle of bonds in $x$-direction. Thus this is a random vector-potential model with a short-range correlator 
$\left\langle A_{\alpha}(i) A_{\beta}(j)\right\rangle=(\Phi / L)^{2} \delta_{\alpha, x} \delta_{\beta, x} \delta_{i j}$ of the type given in the continuous approximation by Eq.(2.6). The only difference is that the correlator is anisotropic. This difference is not important, since it leads only to a constant factor $1 / d$ in $v_{\tau}$ that can be absorbed in the parameter $\phi$. This kind of perturbation is qualitatively different from case I, since it acts locally.

The numerical evaluation of the curvature is based on the representation of the second derivative by the finite difference:

$$
K_{n}=2 \phi^{-2}\left[E_{n}(\phi)-E_{n}(0)\right] .
$$

In using this formula one should take care that $\phi$ is small enough in order for Eq.(5.2) to be valid. On the other hand, a $\phi$ too small would result in big numerical errors because of the finite numerical precision in evaluating $E_{n}$. The optimal choice of $\phi$ should be made for each level $E_{n}$ separately, since the level curvatures vary in a wide range for a given realization of disorder. To this end, we diagonalize the Hamiltonian for several values of $\phi$ (up to ten values) for each disorder realization and choose the smallest $\phi$ for which the normalized difference $\left|E_{n}(\phi)-E_{n}(0)\right| / E_{n}(0)$ is still larger than some conveniently chosen small parameter. In order to attain a smooth curve and decrease the statistical fluctuations the statistical average has been made both over the energies (in the energy window of width 4 centered at $E=0$ ) and over many realizations of the disorder (there were typically few thousands of them).

\section{A. Corrections to $P(K)$ beyond RMT in $3 d$ metals.}

In this section we compute the finite $g$ corrections to the shape of the curvature distribution in the metallic regime, comparing the numerical results with the regular corrections, Eq.(2.1), (2.2).

It turns out that the magnitude of corrections is small and we need to consider a rather large disorder $(W \sim 10)$ to detect it. At yet larger values of disorder we may enter the critical regime. The onset of the critical regime exhibits itself in the weak dependence of the conductance $g$ and the magnitude of correction to $P(k)$ on the system size $L$. In contrast in a good metal a naive estimation of $C_{d}$ in Eq.(2.1) yields $\left|C_{d}\right| \propto W^{4} / L^{2}$. The upper limit of the coefficient $\left|C_{d}\right|$ can be estimated from the critical conductance $g_{3}^{*}=4 \pi^{2} g^{*} \approx 1$. Since $\left|C_{d}\right|$ is proportional to a small parameter $\left(4 \pi^{3} g\right)^{-2}=\left(\pi g_{3}\right)^{-2}$, its value just near the critical region is proportional to a small numerical factor $1 / \pi^{2} \sim 0.1$. It is this small numerical factor together with the strong $W$-dependence of $C_{d}$ that makes the correction to $P(k)$ small and Eq.(2.1) qualitatively applicable even very close to the mobility edge.

\section{Global Vector Potential.}

In Fig. 1 we show the numerical results for $\delta P(k)=P(k)-P_{W D}(k)$ for the $3 d$ metallic regime in case $\mathbf{I}$. The calculations are performed for system size $L=8$ and disorder $W=12$. The number of disorder realizations is 1500 . The deviation of $P(k)$ from the RMT result is very small, less than one percent. The magnitude of the statistical noise present after averaging is done appears to be only a little smaller than the signal itself. Nevertheless the 
general trend of the curve agrees with the analytical prediction Eq.(2.1): $P(k)$ is above the RMT result at small $k$. We have used the coefficient $C_{d}$ in Eq.(2.1) as a free parameter in the least square fitting of the numerical results. The value $C_{3}=0.0044$ found from such a fitting is probably a reliable estimate of the magnitude of the correction $\delta P(k)$ in the above case.

\section{Random Magnetic Flux}

The same correction $\delta P(k)$ for the case of a random magnetic flux is displayed in Fig. 5 . The values of the parameters of the Hamiltonian are the same as for the previous case. Despite the statistical fluctuations are still rather strong, the numerical results are quite significant. We see that again the expression Eq.(2.1) provides a rather good one-parameter fitting function for the numerical results. However, in this case the coefficient $C_{d}=-0.014$ is negative in full agreement with the analytical prediction. Moreover, the numerical results are consistent with the analytical prediction even quantitatively. It follows from Eq.(2.2) that there is a magic relationship for the ratio of amplitudes of the correction in case $\mathbf{I}$ and case II:

$$
R=\frac{C_{3}^{(I)}}{C_{3}^{(I I)}}=-\frac{1}{3},
$$

Our calculations give a result $R=-0.32$ which is in an amazingly good agreement with Eq.(5.3).

\section{B. $P(k)$ at the mobility edge in $3 d$ and in $2 d$ metals.}

A numerical investigation of the distribution $P(k)$ at the Anderson transition critical point has been already carried out in Ref. [10]. The main finding of the numerical simulation is that the distribution function at the mobility edge is remarkably well fitted by the formula:

$$
P_{\alpha}(k)=\frac{A_{\alpha}}{\left(1+|k|^{(2-\alpha)}\right)^{\frac{3}{2-\alpha}}} .
$$

with $\alpha \approx 0.4$. Equation (5.4) defines a function that has a branching point of the type Eq.(4.4) at $k=0$ and the asymptotic behavior $P_{\alpha}(k) \propto|k|^{-3}$, expected in all cases where there is energy level repulsion $R(\omega, 0) \propto|\omega|$ at $\omega \ll 1$. The function $P_{\alpha}(k)$ is a rather special one 10 , since once it is properly normalized by choosing $A_{\alpha}=(2-\alpha) \Gamma[3 /(2-\alpha)]\{[\Gamma[1 /(2-$ $\alpha)] \Gamma(2 /(2-\alpha)]\}^{-1}>1$, it automatically satisfies the condition $\int d k|k| P_{\alpha}(k)=1$ for all $\alpha$. Thus the full distribution $P_{\alpha}(k)$ is determined uniquely by its value at $k=0$. If we take $P_{\alpha}(k=0)$ equal to the numerical result (that is known up to a small error bar) the whole curve is parameter free. Alternatively we can consider $\alpha$ as a free parameter that should be determined by a least square fitting of the overall numerical curve. These two procedures yield very close values for $\alpha$ and an excellent overall fitting of the numerical curve, implying that the extrapolation by means of the function $P_{\alpha}(k)$ is very self-consistent.

In Fig. 6 we plot the results for the difference $\delta P(k)=P(k)-P_{W D}(k)$ for the critical disorder $W=16.5$ and the system size $L=12$ as compared to two one-parameter fitting 
curves provided by Eq.(2.1) and Eq.(5.4). It is clearly seen that despite the analytic function $\delta P_{\text {reg }}(k)$ given by Eq.(2.1) reproduces a correct qualitative behavior of $\delta P(k)$, there is some feature at small $|k|$ that is captured better by the nonanalytic fitting function, Eq.(5.4).

We note also that this enhancement at small $|k|$ relative to $\delta P_{\text {reg }}(k)$ is size-dependent, with $P(k=0)$ increasing with $L$ so that a full saturation is not reached even for $L=10$. Such a behavior is beyond the one-parameter scaling and can be explained by the termination of the power-law tail in the characteristic function $\tilde{P}(\lambda)$ at a finite $\lambda=(L / l)^{4}$. Indeed, the power-law tail given by Eq.(4.3), makes a contribution to $P(k=0)$ proportional to $\int_{1}^{(L / l)^{4}} \lambda^{-\mu} d \lambda$. This contribution is size-dependent and increases with increasing $L$ (with a saturation at $L / l \rightarrow \infty$ ) even exactly at the critical point where the exponent $\mu$ is a constant.

Now let us calculate the fractal dimension $d_{2}=3-\eta$ using the relationship, Eq.(4.8), between $\eta$ and $\mu=3-\alpha$. For $\alpha \approx 0.4$ we have $\mu \approx 2.6$ and $d_{2} \approx 1.5$. This value is in a good agreement with direct evaluation $\left.\right|_{0}$ of $d_{2}$ from Eqs.(14.5),(4.6).

We also checked that in a $2 d$ metal the deviation from the RMT result $\delta P(k)$ has the same qualitative form as in the $3 d$ critical case. In Fig. 0 we present plots of $\delta P(k)$ for different values of $L$ and $W=6$. An interesting feature seen in the figure is the fixed point in $\delta P(k)$ at $k \approx 0.35$. Despite the one-parameter function $P_{\alpha}(k)$ does not have an exact fixed point at the same value of $k$, all the curves obtained varying $\alpha$ do get very close to each other at $k \approx 0.35$ in what looks almost like a fixed point. As in the $3 d$ critical case, the analytic function $\delta P_{\text {reg }}(k)$ fits well the overall distribution but fails to describe the sharp enhancement for small curvatures that is instead well described by the function $P_{\alpha}(k)$. This is shown in Fig. 8 for the system $L=30, W=6$.

\section{CONCLUSIONS}

In this paper we have investigated both analytically and numerically the relationship between the statistics of eigenfunctions and the spectral statistics in disordered conductors. The level curvature distribution has been chosen as the target of our study, since it is the simplest known example of parametric spectral statistics that can be used as a spectral probe of the structure of eigenfunctions.

The main results of the paper are formulated in Eqs.(2.1),(2.2), and Eqs.(4.4),(4.8). Numerical results in agreement with these analytical predictions are presented in Figs. 0.5, and 6.8.8 respectively. The first two equations describe the regular corrections beyond RMT to the level curvature distribution in disordered metals. These corrections stem from long-range correlations in the wave functions with a typical length scale of the order of the sample size. The latter two equations summarize the effect of local irregularities (sharp peaks) in the structure of eigenfunctions in its most developed form (multifractality) in the critical region near the mobility edge.

Moreover, Eq.(4.8) suggests an explicit relationship between the fractal dimension $d_{2}=d-\eta$ of a critical eigenfunction and the exponent $\mu$ in the power-law tail Eq.(1.3) of the characteristic function $\tilde{P}_{c}(\lambda)$ that describes the particular parametric spectral statistics considered here (level curvature distribution).

Note that Eq.(4.8) is more general than Eq.(4.4). The latter requires rather strong multifractality $\eta>\frac{4}{3}$, while the former applies to a generic critical state. For instance, it 
would be interesting to check its validity for the critical state in the quantum Hall effect, where $\eta \approx 0.5$ and we predict $\mu \approx 8$. Recent progress 41 in numerical simulations on the Chalker-Coddington network model ${ }^{42}$ seems to make the task attainable.

As far as the regular corrections are concerned, there is an interesting question of what happens to them for $d>4$. The sum in Eq.(2.2) is a parameter-free number only for $d<4$ when it converges. For $d>4$ the sum is divergent and requires a cut-off at large $|\mathbf{q}|$. Thus for the correct evaluation of this sum it is necessary to go beyond the diffusion approximation and the approximation of slow spatial variations of the field $Q(\mathbf{r})$ in the nonlinear sigma-model. The divergent sum in Eq.(2.2) implies that for $d>4$ the correction $\delta P(k)$ to the level curvature distribution is dominated by short-range spatial correlations of the eigenfunctions, in contrast to $d<4$ where it is dominated by long-range correlations. Based on our discussion at the end of Sec. IIA, the short-range nature of the eigenfunction correlations in $d>4$ is most likely the cause of the change of sign in the correction $\delta P(k)$ for the "global" case I (as compared to $d<4$ ) and the reason why $\delta P(k)$ shows qualitatively the same behavior of case II ("local" perturbations).

One of the most important results Eq. 2.4) of our calculations is that the ratio $r(g)$ of the mean level curvature $\langle|K|\rangle$ and the mean Drude conductance $2 g$ is not a constant and is always larger than the RMT result $r=1$. This is in a qualitative agreement with the result 43,4$]$ that $\langle|K|\rangle \propto \sqrt{g}$ in a strictly one-dimensional case where $g \ll 1$ and localization effects are strong. Indeed, let us assume that the square-root dependence is typical for strongly localized states in any dimensions. Then the function $r(g)$ should behave like $r(g) \propto g^{-1 / 2}$ at small $g \ll 1$ and $r(g) \rightarrow 1$ for $g \rightarrow \infty$. If in addition we make the natural assumption that $r(g)$ is a monotonic function, we arrive at the conclusion that $\delta r(g)>0$ everywhere in agreement with Eq.(2.4).

A similar deviation from the proportionality relationship $\langle|K|\rangle \propto g$ has been observed recently in numerical simulations 19.

\section{Acknowledgements}

We thank B.L.Altshuler, E.Akkermans, V.I.Fal'ko, Y.V.Fyodorov, I.V.Lerner, A.D.Mirlin, W. Stephan and $\mathrm{Yu} \mathrm{Lu}$ for stimulating discussions. V.E.K. is grateful for the hospitality extended to him at the Newton Institute (Cambridge, UK) where the final part of this work has been completed. Support from grants RFBR/INTAS No.95-675, CRDF No.RP1209 (V.E.K.) and EPSRC grant No.GR/K95505 (I.V.Y.) is also gratefully acknowledged. C.M.C. thanks the Swedish NFR and TFR for financial support.

\section{APPENDIX A: EFFECTIVE ACTION}

The parametrization (2.13) enables us to single out fast modes $\widetilde{Q}$ in the action (2.12) as follows:

$$
F\left[\widetilde{Q}, Q_{0}\right]=\operatorname{Str}\left\{\frac{\pi g}{8}\left(\left[\vec{Q}_{\phi}, \widetilde{Q}\right]-i L \nabla \widetilde{Q}\right)^{2}-\left(i \frac{\pi g}{4} k \phi^{2} Q_{\Lambda}+Q_{J}\right) \widetilde{Q}\right\},
$$

where the following notation has been used:

$$
\vec{Q}_{\phi}=T_{0} \widehat{\phi} T_{0}^{-1}, \quad Q_{\Lambda}=T_{0} \Lambda T_{0}^{-1}, \quad Q_{J}=T_{0} J T_{0}^{-1} .
$$




$$
g=\frac{D}{\Delta L^{2}}=\nu D L^{d-2}, \quad S \operatorname{Tr} \ldots \equiv \int \frac{d \mathbf{r}}{V} \operatorname{Str} \ldots, \quad K=2 g \cdot k .
$$

Taking advantage of the fact that we are interested in $\phi \rightarrow 0$ limit only, it is convenient to absorb the large conductance into the definition of the flux $\frac{\pi}{2} g \phi^{2} \rightarrow \phi^{2}$ (wherever possible). Then introducing the dimensionless gradient $\partial=\sqrt{\pi / 2} L \nabla$ and using the identity

$$
\frac{\phi^{2}}{2} \operatorname{STr} Q_{\Lambda} \widetilde{Q}=S \operatorname{Tr} \vec{Q}_{\phi}^{2} \widetilde{Q}
$$

we recast the action in the following form

$$
F\left[\widetilde{Q}, Q_{0}\right]=S T r\left\{\frac{1}{4}\left(\left[\vec{Q}_{\phi}, \widetilde{Q}\right]-i \sqrt{g} \partial \widetilde{Q}\right)^{2}-\left(i k \vec{Q}_{\phi}^{2}+Q_{J}\right) \widetilde{Q}\right\} .
$$

These manipulations, along with fact that $\widetilde{W}$ scales as $\widetilde{W}=g^{-1 / 2} w, w \propto 1$ enable us to construct a perturbative expansion in the parameter $1 / \mathrm{g}$-straightforwardly, simply expanding $F\left[\widetilde{Q}, Q_{0}\right]$ in powers of $w$. This procedure is equivalent to selecting diagrams contributing to the same order in $1 / \mathrm{g}$. Then for the partition function we get the representation:

$$
Z=\lim _{\phi \rightarrow 0} \frac{-\phi^{2}}{32 \pi g} \Re \int D Q_{0} D \widetilde{Q} \cdot J[\widetilde{Q}] \exp \left(-F\left[\widetilde{Q}, Q_{0}\right]\right)
$$

where $J[\widetilde{Q}]$ is the Jacobian of the transformation $Q \rightarrow\left(\widetilde{Q}, Q_{0}\right)$ (obtained in the following Appendix $\mathrm{B}$ ). The action ( $\mathrm{A2})$ is expanded as follows:

$$
F\left[\widetilde{Q}, Q_{0}\right]=F\left[Q_{0}\right]+\frac{1}{4} S \operatorname{Tr}(\partial w)^{2}+\sum_{n=1}^{\infty} g^{-n / 2} F_{n / 2}\left[Q_{0}, w\right] .
$$

The first term of the expansion is nothing but the zero-mode action ( $R M T$-limit):

$$
F\left[Q_{0}\right]=\operatorname{STr}\left[\frac{1}{2}\left(\vec{Q}_{\phi} \Lambda\right)^{2}-\left(i k \vec{Q}_{\phi}^{2}+Q_{J}\right) \Lambda\right] .
$$

The second term in (A4) corresponds to the noninteracting diffusion modes approximation. The other terms in ( $\overline{\mathrm{A} 4}$ ) describe the interaction of the diffusion and zero modes with $g^{-1}$ playing the role of a coupling constant. For our purposes it is enough to keep the first four interaction terms of expansion:

$$
\begin{gathered}
F_{1 / 2}=i S \operatorname{Tr} \vec{Q}_{\phi} w \partial w \\
F_{1}=\frac{1}{2} \operatorname{STr}\left\{A \Lambda w^{2}+\left(\vec{Q}_{\phi} \Lambda w\right)^{2}+\frac{1}{4}(\partial w)^{2} w^{2}\right\}+ \\
+\frac{1}{8 i} \operatorname{STr}\left\{\vec{Q}_{\phi}\left(\partial w^{3}-2 w \cdot \partial w \cdot w\right)\right\} \\
F_{3 / 2}=\frac{1}{4} \operatorname{STr}\left\{A \Lambda w^{3}+2\left(\vec{Q}_{\phi} \Lambda w\right)^{2} w\right\}+ \\
+\frac{1}{4 i} \operatorname{STr}\left\{\vec{Q}_{\phi}\left(\partial w^{3} \cdot w+\partial w \cdot w^{3}+\partial w^{2} \cdot w^{2}\right)\right\}
\end{gathered}
$$




$$
\begin{aligned}
F_{2} & =\frac{1}{8} \operatorname{STr}\left\{A \Lambda w^{4}+\left(\vec{Q}_{\phi} \Lambda w^{2}\right)^{2}+2\left(\vec{Q}_{\phi} \Lambda w\right)^{2} w^{2}+\frac{1}{2 i} \vec{Q}_{\phi} \partial w^{5}\right\}, \\
& +\frac{1}{64} \operatorname{STr}\left\{\left(\partial w^{3}\right)^{2}+2 \partial w^{5} \cdot \partial w-2 \partial w^{4} \cdot \partial w^{2}\right\} .
\end{aligned}
$$

We have used the notation:

$$
A=\vec{Q}_{\phi} \Lambda \vec{Q}_{\phi}-i k \vec{Q}_{\phi}^{2}-Q_{J}
$$

Averaging over the fast modes can be implemented by the use of contraction rules derived in 36 . We recast them in the following form:

$$
\begin{aligned}
& \left\langle\widetilde{W}(\mathbf{r}) R \widetilde{W}\left(\mathbf{r}^{\prime}\right)\right\rangle=\Pi\left(\mathbf{r}, \mathbf{r}^{\prime}\right)\{S \operatorname{Tr} R-S \operatorname{Tr} \Lambda R \Lambda-\bar{R}+\Lambda \bar{R} \Lambda\}, \\
& \left\langle\widetilde{W}(\mathbf{r}) \operatorname{STr} R \widetilde{W}\left(\mathbf{r}^{\prime}\right)\right\rangle=\Pi\left(\mathbf{r}, \mathbf{r}^{\prime}\right)\{R-\bar{R}-\Lambda(R-\bar{R}) \Lambda\} .
\end{aligned}
$$

The propagator $\Pi\left(\mathbf{r}, \mathbf{r}^{\prime}\right)$ satisfies the diffusion equation:

$$
-\Delta \Pi\left(\mathbf{r}, \mathbf{r}^{\prime}\right)=\frac{1}{\pi g L^{2}} \cdot \delta\left(\mathbf{r}-\mathbf{r}^{\prime}\right), \quad \Pi^{-1}(\mathbf{q})=\pi g(\mathbf{q} L)^{2} .
$$

The contraction rules (A10) provide a basis for integrating out the fast modes in (A3). For example, straightforward but lengthy calculations give us the following rules for the integration of product of two vertices from (A7) containing no gradients:

$$
\begin{aligned}
& \left\langle S \operatorname{Sr} A \Lambda \widetilde{W}^{2}(\mathbf{r}) S \operatorname{Tr} A \Lambda \widetilde{W}^{2}\left(\mathbf{r}^{\prime}\right)\right\rangle_{c}=4 \Pi^{2}\left(\mathbf{r}, \mathbf{r}^{\prime}\right)\left\{[S \operatorname{Tr} A \Lambda]^{2}-[S \operatorname{Tr} A]^{2}\right\}, \\
& \left\langle S \operatorname{ST}[B \Lambda \widetilde{W}(\mathbf{r})]^{2} S \operatorname{ST}\left[B \Lambda \widetilde{W}\left(\mathbf{r}^{\prime}\right)\right]^{2}\right\rangle_{c}=4 \Pi^{2}\left(\mathbf{r}, \mathbf{r}^{\prime}\right) \cdot\left\{\left[S \operatorname{Sr}(B \Lambda)^{2}\right]^{2}+\right. \\
+ & {\left.\left[S \operatorname{ST} B^{2}\right]^{2}-2\left[S \operatorname{Tr} B^{2} \Lambda\right]^{2}+S \operatorname{Sr}\left((B \Lambda)^{4}+B^{4}-2\left(B^{2} \Lambda\right)^{2}\right)\right\}, } \\
& \left\langle S \operatorname{ST}[B \Lambda \widetilde{W}(\mathbf{r})]^{2} S \operatorname{ST} A \Lambda \widetilde{W}^{2}\left(\mathbf{r}^{\prime}\right)\right\rangle_{c}= \\
= & 4 \Pi^{2}\left(\mathbf{r}, \mathbf{r}^{\prime}\right) \cdot S \operatorname{Sr} A\left(2 B^{2} \Lambda-B \Lambda B-\Lambda(B \Lambda)^{2}\right) .
\end{aligned}
$$

where the matrices satisfy the symmetry relations $\bar{A}=A, \quad \bar{B}=-B$ (for notations see国) and the brackets $\langle\ldots\rangle_{c}$ mean keeping only the connected parts of the correlators after averaging over fast modes $\widetilde{W}$.

These calculations can be easily extended to couplings of gradient vertices as well. Then having at hand all possible couplings we are able to construct a cumulant expansion for the partition function (A3). Keeping all nonvanishing correlators up to the second order in $1 / g$ we arrive at:

$$
\begin{gathered}
F^{e f f}=F_{0}+\left\langle F_{1}\right\rangle-\frac{1}{2}\left\langle F_{1}^{2}\right\rangle_{c}+\frac{1}{2}\left\langle F_{1} F_{1 / 2}^{2}\right\rangle_{c}-\frac{1}{4 !}\left\langle F_{1 / 2}^{4}\right\rangle_{c}, \\
F_{1}^{e f f}=\left\langle F_{1}\right\rangle=-\frac{1}{2} \sum_{\mathbf{q}} \Pi(\mathbf{q}) \cdot S \operatorname{ST}\left(\vec{Q}_{\phi} \Lambda\right)^{2}, \\
F_{2}^{e f f}=-\frac{1}{2}\left\langle F_{1}^{2}\right\rangle_{c}+\frac{1}{2}\left\langle F_{1} F_{1 / 2}^{2}\right\rangle_{c}-\frac{1}{4 !}\left\langle F_{1 / 2}^{4}\right\rangle_{c}=4 \sum_{\mathbf{q}} \Pi^{2}(\mathbf{q}) \cdot\left(f_{2}+f_{3}+f_{4}\right) .
\end{gathered}
$$


Here the term $f_{2}$ corresponds to coupling of two gradientless vertices (A7):

$$
\begin{aligned}
& -8 f_{2}=[S \operatorname{ST} A \Lambda]^{2}-[S \operatorname{Tr} A]^{2}+\left[S \operatorname{ST}\left(\vec{Q}_{\phi} \Lambda\right)^{2}\right]^{2}-2\left[S \operatorname{ST} \vec{Q}_{\phi}^{2} \Lambda\right]^{2} \\
& -\operatorname{STr}\left\{\left(\vec{Q}_{\phi} \Lambda\right)^{4}-2\left(\vec{Q}_{\phi}^{2} \Lambda\right)^{2}+2 A\left(2 \vec{Q}_{\phi}^{2} \Lambda-\vec{Q}_{\phi} \Lambda \vec{Q}_{\phi}-\Lambda\left(\vec{Q}_{\phi} \Lambda\right)^{2}\right)\right\},
\end{aligned}
$$

$f_{3}$ describes the coupling of two gradient vertices (A5) to the gradientless one(A7):

$$
\begin{aligned}
2 d \cdot f_{3}= & S \operatorname{Tr} A \Lambda \cdot S \operatorname{Tr}\left(\vec{Q}_{\phi} \Lambda\right)^{2}-2 S \operatorname{Tr} A \cdot S \operatorname{Tr} \vec{Q}_{\phi}^{2} \Lambda+\left[S \operatorname{Tr}\left(\vec{Q}_{\phi} \Lambda\right)^{2}\right]^{2} \\
& -4\left[S \operatorname{Sr} \vec{Q}_{\phi}^{2} \Lambda\right]^{2}+2 S \operatorname{Tr}\left(\vec{Q}_{\phi}^{2} \Lambda\right)^{2},
\end{aligned}
$$

and the last contribution $f_{4}$ comes from 4 coupled gradient vertices (A5):

$$
-\frac{4}{9} d(d+2) \cdot f_{4}=\left[S \operatorname{ST}\left(\vec{Q}_{\phi} \Lambda\right)^{2}\right]^{2}-4\left[S \operatorname{Sr} \vec{Q}_{\phi}^{2} \Lambda\right]^{2}
$$

The expression for $F^{e f f}$ can be simplified considerably if one uses the following factorization properties of long traces:

$$
\begin{aligned}
& \operatorname{Str}\left[\left(\hat{\phi}^{2} Q_{0}\right)^{2}\right]=\left[\operatorname{Str}\left[\hat{\phi}^{2} Q_{0}\right]\right]^{2}=\beta^{2} \\
& \operatorname{Str}\left[\left(\hat{\phi} Q_{0}\right)^{4}\right]=\frac{1}{2}\left[\operatorname{Str}\left[\left(\hat{\phi} Q_{0}\right)^{2}\right]\right]^{2}=\frac{\alpha^{2}}{2} \\
& \operatorname{Str}\left[\left(\hat{\phi} Q_{0}\right)^{2} \hat{\phi}^{2} Q_{0}\right]=\frac{1}{2} \operatorname{Str}\left[\left(\hat{\phi} Q_{0}\right)^{2}\right] \operatorname{Str}\left[\hat{\phi}^{2} Q_{0}\right]=\frac{\alpha \beta}{2} \\
& \operatorname{Str}\left[\left(\hat{\phi} Q_{0}\right)^{2} \hat{k} Q_{0}^{p p}\right]=\frac{1}{2} \operatorname{Str}\left[\left(\hat{\phi} Q_{0}\right)^{2}\right] \operatorname{Str}\left[\hat{k} Q_{0}^{p p}\right]=\frac{\alpha}{2} \operatorname{Str}\left[\hat{k} Q_{0}^{p p}\right] .
\end{aligned}
$$

The factorization holds in the leading power in the noncompact angles in the original Efetov's parametrization and can be shown by straightforward but extremely lengthy calculations.

It is worth noting that the Jacobian $J[\widetilde{Q}]$ also contributes to the effective action (the last term in Eq.(2.19)). Taking into account Eq.(B10) of Appendix B one concludes that the Jacobian leads to the replacement $\mathbf{q}^{2}+\frac{1}{\pi g}$ in $\Pi(\mathbf{q})$. Thus the $1 / g^{2}$ contribution from the Jacobian follows from the correction to $\Pi(\mathbf{q})$ in $F_{1}^{e f f}$ :

$$
F_{j a c o b}^{e f f}=\frac{1}{2} \sum_{\mathbf{q}} \Pi(\mathbf{q}) \cdot S \operatorname{Tr}\left(\vec{Q}_{\phi} \Lambda\right)^{2}
$$

Collecting all the results obtained in this Appendix we arrive at the effective action $\mathcal{F}\left[Q_{0}\right]$ given in Eqs.(2.19),(2.20).

Using these equations one can represent $P(k)$ in the differential form of Eq.(2.22). It turns out that all the relevant derivatives of $P_{a}(k)$ over $k$ can be expressed through the derivatives over $a$. Using the following identities: 


$$
\begin{aligned}
& \left.k \frac{\partial}{\partial k} P_{a}(k)\right|_{a=1}=-\left.\left(1+\frac{\partial}{\partial a}\right) P_{a}(k)\right|_{a=1} ; \\
& \left.k \frac{\partial^{2}}{\partial k \partial a} P_{a}(k)\right|_{a=1}=-\left.\left(\frac{\partial^{2}}{\partial a^{2}}+2 \frac{\partial}{\partial a}\right) P_{a}(k)\right|_{a=1} ; \\
& \left.\frac{\partial^{2}}{\partial k^{2}} P_{a}(k)\right|_{a=1}=\left.\left(\frac{\partial}{\partial a}-\frac{\partial^{2}}{\partial a^{2}}\right) P_{a}(k)\right|_{a=1} ; \\
& \left.k^{2} \frac{\partial^{2}}{\partial k^{2}} P_{a}(k)\right|_{a=1}=\left.\left(2+4 \frac{\partial}{\partial a}+\frac{\partial^{2}}{\partial a^{2}}\right) P_{a}(k)\right|_{a=1}
\end{aligned}
$$

we can finally represent $\delta P(k)$ in the form Eq. 2.24) that contains only the first and second derivatives with respect of $a$.

\section{APPENDIX B: CALCULATION OF THE JACOBIAN}

In the evaluation of the Jacobian of the transformation $Q \rightarrow\left(Q_{0}, \widetilde{Q}\right)$ we follow the procedure proposed in Ref. [24] and prove that the Jacobian does not depend on zero-mode $Q_{0} 37$. The derivation is simplified if we go to the "rational" parametrization first:

$$
Q=(1-W) \Lambda(1-W)^{-1}, \quad W=\left(\bar{B}^{B}\right) .
$$

This parametrization has been known since Efetov's work 0 . The advantage of this representation lies in the fact that Jacobian of the transformation $Q \rightarrow W$ equals to unity. On the another hand we have the decomposition (2.13). Taking into account that $T_{0}$ belongs to the graded coset space $\operatorname{UOSP}(2,2 \mid 4) / \operatorname{UOSP}(2 \mid 2) \otimes U O S P(2 \mid 2)$ the complete parametrization takes the form:

$$
\begin{aligned}
Q & =T_{0}^{-1} \widetilde{Q} T_{0}, \quad T_{0}=\sqrt{\frac{1+W_{0}}{1-W_{0}}}, \quad \widetilde{Q}=(1-\widetilde{W}) \Lambda(1-\widetilde{W})^{-1}, \\
W_{0} & =\left(\text { ( }_{0} B_{0}\right), \quad \widetilde{W}=\left({ }_{\widetilde{B}}^{\widetilde{B}}\right) .
\end{aligned}
$$

Comparing these two representations $(\overline{\mathrm{B} 1})$ and $(\overline{\mathrm{B} 2})$ we derive the connection between $W$ and $\left(W_{0}, \widetilde{W}\right)$ :

$$
\begin{gathered}
W=\left(W_{0}+\varpi\right)\left(1+W_{0} \varpi\right)^{-1}, \quad \varpi=\left(1-W_{0}^{2}\right)^{-1 / 2} \widetilde{W}\left(1-W_{0}^{2}\right)^{1 / 2}, \\
B=\left(B_{0}+b\right)\left(1+B_{0} b\right)^{-1}, \quad b=\left(1-B_{0} \bar{B}_{0}\right)^{-1 / 2} \widetilde{B}\left(1-\bar{B}_{0} B_{0}\right)^{1 / 2} .
\end{gathered}
$$

Since the field $b$ (being proportional to $\widetilde{W}$ ) contains only nonzero momenta it can be treated perturbatively. Going to the Fourier representation and expanding (B3) up to second order in $b$ we separate zero- and nonzero momenta:

$$
\begin{aligned}
& B(\mathbf{k}=0)=B_{0}-S b_{\mathbf{q}} \bar{B}_{0} b_{-\mathbf{q}}, \\
& B(\mathbf{k} \neq 0)=S\left(b_{\mathbf{k}}-b_{\mathbf{k}+\mathbf{q}} \bar{B}_{0} b_{-\mathbf{q}}+b_{\mathbf{k}+\mathbf{q}_{1}+\mathbf{q}_{2}} \bar{B}_{0} b_{-\mathbf{q}_{1}} \bar{B}_{0} b_{-\mathbf{q}_{2}}\right),
\end{aligned}
$$


where $S=\left(1-B_{0} \bar{B}_{0}\right)$ has been introduced and the summation over repeated indecis is implied. Therefore, we are interested in Jacobian of the transformation $B \rightarrow\left(B_{0}, b\right)$ which is equivalent to $(B(\mathbf{k}=0), B(\mathbf{k} \neq 0)) \rightarrow\left(B_{0}, b_{\mathbf{k} \neq 0}\right)$. The corresponding Jacobian can be represented in the block-matrix structure:

$$
J=S \operatorname{Det}\left(\begin{array}{ll}
\partial B(0) / \partial B_{0} & \partial B(0) / \partial b \\
\partial B(\emptyset) / \partial B_{0} & \partial B(\emptyset) / \partial b
\end{array}\right)
$$

where a short notation for the following supermatrices has been introduced:

$$
\begin{aligned}
{[\partial B(0) / \partial b]_{0 k} } & =\partial B(q=0) / \partial b_{k}, \\
{\left[\partial B(\emptyset) / \partial B_{0}\right]_{k 0} } & =\partial B(k \neq 0) / \partial B_{0}, \\
{[\partial B(\emptyset) / \partial b]_{k k^{\prime}} } & =\partial B(k \neq 0) / \partial b_{k^{\prime}},
\end{aligned}
$$

and right derivatives are implied $\mathrm{E}$. Using the identity for the superdeterminant of block matrices we recast (B5) in the following form

$$
\begin{aligned}
J & =J_{1} \cdot J_{2}, \quad J_{1}=S \operatorname{Det} \frac{\partial B(\emptyset)}{\partial b}, \\
J_{2} & =S \operatorname{det}\left[\frac{\partial B(0)}{\partial B_{0}}-\frac{\partial B(0)}{\partial b_{k}}\left(\frac{\partial B(\emptyset)}{\partial b}\right)_{k k^{\prime}}^{-1} \frac{\partial B\left(k^{\prime} \neq 0\right)}{\partial B_{0}}\right],
\end{aligned}
$$

where $S$ det acts within the space of $4 \times 4$ matrices, while $S$ Det spans $k$-space also. Lengthy but straightforward calculations give us following very useful formulae:

$$
\begin{gathered}
\operatorname{Str} \frac{\partial(D b F)}{\partial b}=\operatorname{Str} D \cdot \operatorname{Str} F, \quad \operatorname{Str} \frac{\partial(D \bar{b} F)}{\partial b}=\operatorname{Str} D \bar{F} \\
\operatorname{Str} \frac{\partial\left(D_{1} b_{1} F_{1}\right)}{\partial b_{1}} \frac{\partial\left(D_{2} b_{2} F_{2}\right)}{\partial b_{2}}=\operatorname{Str} D_{1} D_{2} \cdot \operatorname{Str} F_{1} F_{2}, \\
\operatorname{Str} \frac{\partial\left(D_{1} b_{1} F_{1}\right)}{\partial b_{1}} \frac{\partial\left(D_{2} \bar{b}_{2} F_{2}\right)}{\partial b_{2}}=\operatorname{Str} D_{1} D_{2} \bar{F}_{1} \bar{F}_{2}, \\
\operatorname{Str} \frac{\partial\left(D_{1} b_{1} F_{1}\right)}{\partial b_{1}}\left[\frac{\partial(G b)}{\partial b}\right]^{-1} \frac{\partial\left(D_{2} \bar{b}_{2} F_{2}\right)}{\partial b_{2}}=S \operatorname{St} D_{1} G^{-1} D_{2} \bar{F}_{1} \bar{F}_{2} .
\end{gathered}
$$

Now we expand $J_{1}$ up to second order in $b$ :

$$
J_{1}=S \operatorname{Det} \frac{\partial(S b)}{\partial b} \cdot K
$$

where

$$
K=\exp \left\{S \operatorname{Tr}\left(\frac{\partial(S b)}{\partial b}\right)^{-1} \frac{\partial\left(b \bar{B}_{0} b \bar{B}_{0} b\right)}{\partial b}-\frac{1}{2} S \operatorname{Tr}\left[\left(\frac{\partial(S b)}{\partial b}\right)^{-1} \frac{\partial\left(S b \bar{B}_{0} b\right)}{\partial b}\right]^{2}\right\}
$$




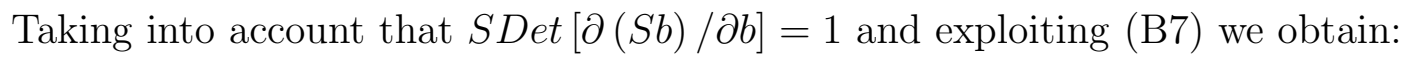

$$
\begin{gathered}
\operatorname{Str}\left(\frac{\partial(S b)}{\partial b}\right)^{-1} \frac{\partial\left(S b \bar{B}_{0} b \bar{B}_{0} b\right)}{\partial b}=\sum_{\mathbf{q} \neq 0, \mathbf{k}} \operatorname{Str} b_{\mathbf{q}} \bar{B}_{0} S \operatorname{tr} \bar{B}_{0} b_{-\mathbf{q}}, \\
\operatorname{Str}\left[\left(\frac{\partial(S b)}{\partial b}\right)^{-1} \frac{\partial\left(S b \bar{B}_{0} b\right)}{\partial b}\right]^{2}=2 \sum_{\mathbf{k}^{\prime} \neq 0, \mathbf{k} \neq 0} \operatorname{Str}_{\mathbf{k}^{\prime}-\mathbf{k}} \bar{B}_{0} S \operatorname{str} \bar{B}_{0} b_{\mathbf{k}-\mathbf{k}^{\prime}}
\end{gathered}
$$

Finally, we arrive at:

$$
J_{1}=\exp \left\{\sum_{\mathbf{k}} S t r b_{-\mathbf{k}} \bar{B}_{0} S t r \bar{B}_{0} b_{\mathbf{k}}\right\} .
$$

Then with the same accuracy we may recast $J_{2}$ in the following form:

$$
\begin{aligned}
J_{2} & =S \operatorname{det}\left(1-\delta J_{2}\right), \\
\delta J_{2} & =\frac{\partial\left(S b_{\mathbf{q}} \bar{B}_{0} b_{-\mathbf{q}}\right)}{\partial B_{0}}-\frac{\partial\left(S b_{\mathbf{q}} \bar{B}_{0} b_{-\mathbf{q}}\right)}{\partial b_{\mathbf{k}}}\left(\frac{\partial(S b)}{\partial b}\right)^{-1} \frac{\partial\left(S b_{\mathbf{k}}\right)}{\partial B_{0}} \\
-\ln J_{2} & \approx S \operatorname{tr} \frac{\partial\left(S b_{\mathbf{q}} \bar{B}_{0} b_{-\mathbf{q}}\right)}{\partial B_{0}}-S \operatorname{tr} \frac{\partial\left(S b_{\mathbf{q}} \bar{B}_{0} b_{-\mathbf{q}}\right)}{\partial b_{\mathbf{k}}}\left(\frac{\partial(S b)}{\partial b}\right)^{-1} \frac{\partial\left(S b_{\mathbf{k}}\right)}{\partial B_{0}}
\end{aligned}
$$

Using formulae (B7) we obtain:

$$
\begin{aligned}
& \operatorname{Str} \frac{\partial\left(S b_{\mathbf{q}} \bar{B}_{0} b_{-\mathbf{q}}\right)}{\partial B_{0}}=\operatorname{Str} S b_{\mathbf{q}} \bar{b}_{-\mathbf{q}}-S \operatorname{tr} \bar{B}_{0} b_{\mathbf{q}} \bar{B}_{0} b_{-\mathbf{q}} \\
& \quad \operatorname{Str} \frac{\partial\left(S b_{\mathbf{q}} \bar{B}_{0} b_{-\mathbf{q}}\right)}{\partial b_{\mathbf{k}}}\left(\frac{\partial(S b)}{\partial b}\right)^{-1} \frac{\partial\left(S b_{\mathbf{k}}\right)}{\partial B_{0}} \\
& =-S \operatorname{tr} S b_{-\mathbf{k}}\left(1-\bar{B}_{0} B_{0}\right)^{-1} \bar{b}_{\mathbf{k}}-S \operatorname{tr} \bar{B}_{0} b_{\mathbf{k}} S \operatorname{tr} \bar{B}_{0} b_{-\mathbf{k}} \\
& +\operatorname{Str} S b_{-\mathbf{k}} \bar{b}_{\mathbf{k}}-\operatorname{Str} \bar{B}_{0} b_{\mathbf{k}} \bar{B}_{0} b_{-\mathbf{k}}
\end{aligned}
$$

As a result we find for the second contribution $J_{2}$ :

$$
\begin{aligned}
-\ln J_{2} & \approx \operatorname{Str}\left(1-B_{0} \bar{B}_{0}\right) b_{-\mathbf{k}}\left(1-\bar{B}_{0} B_{0}\right)^{-1} \bar{b}_{\mathbf{k}}+\operatorname{Str} \bar{B}_{0} b_{\mathbf{k}} \operatorname{Str} \bar{B}_{0} b_{-\mathbf{k}} \\
& =\frac{1}{2} \int \frac{d r}{V} \operatorname{Str} \widetilde{W}^{2}+\operatorname{Str} \bar{B}_{0} b_{\mathbf{k}} \operatorname{Str} \bar{B}_{0} b_{-\mathbf{k}}
\end{aligned}
$$

The parametrization used in this Appendix differs slightly from the one used in the main body of paper. To come back to the original parametrization we must substitute $\widetilde{W} \rightarrow \widetilde{W} / 2$ - Then collecting contributions from $J_{1}$ and $J_{2}$ together we finally obtain Jacobian in the form:

$$
J[\widetilde{Q}]=\exp \left[-\frac{1}{8} \int \frac{d r}{V} S t r \widetilde{W}^{2}\right] .
$$

As one can see from Eq. (B10) the Jacobian does not contain the zero-modes at all. Morover, being quadratic, it just plays the role of a small frequency $(\sim 1 / g)$ in the free diffusion propagator. Expanding such modified propagator we recover the Jacobian contribution to the effective action Eq. (A14). 


\section{APPENDIX C: SOLUTION TO $2 D$ LIOUVILLE EQUATION}

On the boundary of the square $\Omega$ [see Fig. 3] the function $u(z)$ and its first derivative must be continuous. Because, by contruction, $u(x)$ is anti-symmetric when reflected around the sides of $\Omega$, it must be zero on the boundary of $\Omega$ :

$$
\left|\frac{d F}{d z}\right|^{2}=\frac{\gamma^{2}}{16}\left(1+|F(z)|^{2}\right)^{2}
$$

For the case $\gamma \rightarrow 0$ we look for a solution $|F(z)| \gg 1$. Then we have

$$
\frac{d}{d z}\left(\frac{1}{F(z)}\right)=\frac{\gamma}{4} \exp (i \Theta), \quad \operatorname{Im} \Theta=0 .
$$

Suppose we manage to find $\Theta(z)$ which is an analytic function inside the square $\Omega$ and is real on its boundary. Then the equation for $1 / F$ is trivially reducible to quadratures and the solution $F(z)$ is an analytic function inside the square $\Omega$. Then in the limit $\gamma \ll 1$ the solution to the Liouville equation that obeys the condition $u(z)=0$ on the boundary of the square $\Omega$ is found by substituting the function $F(z)$ into Eq.(3.26). In particular, in the region where $|F(z)| \gg 1$ we have:

$$
e^{|u(z)|}=\left|e^{i \Theta(z)}\right|^{2}
$$

Consider the function

$$
z(t)=\frac{\sqrt{2}}{\pi} \int_{0}^{t} \frac{d \xi}{\left(1-\xi^{4}\right)^{1 / 4}}
$$

which does the conformal transformation of the unit circle in the complex plane of $t$ onto the square $\Omega$ in the complex plane of $z$. This function obeys the symmetry property:

$$
z(i t)=i z(t), \quad z\left(t^{*}\right)=z^{*}(t)
$$

If we choose $e^{i \Theta(z)}=[t(z)]^{-k}$, where $k$ is a real parameter, the analytic function $\Theta(z)$ will automatically be real on the boundary of the square $\Omega$. Integrating Eq. (C2) we arrive at:

$$
\frac{1}{F(z)}=\frac{\gamma}{4} \int_{0}^{z}\left[t\left(z^{\prime}\right)\right]^{-k} d z^{\prime}
$$

For $|z| \ll 1$ we have from Eq.(C4):

$$
z=\frac{\sqrt{2}}{\pi} t
$$

and

$$
F(z)=-\frac{4}{\gamma}\left(\frac{\pi}{\sqrt{2}}\right)^{k}(k-1) z^{k-1}, \quad(|z| \ll 1) .
$$

We see that, indeed, $|F(z)| \gg 1$ for 


$$
|z|>r_{0}=\frac{\sqrt{2}}{\pi}\left[\frac{\gamma}{2 \pi \sqrt{2}(k-1)}\right]^{\frac{1}{k-1}}, \quad(k>1) .
$$

In this region the function $u(z)$ is given by:

$$
u(z)=-2 k \ln |t(z)|=-2 k \Re \ln t(z), \quad\left(|z| \gg r_{0}\right) .
$$

Thus for $r_{0} \ll 1$, the solution to the $2 d$ Liouville equation with boundary conditions $u(z)=0$ on the boundary of the square $\Omega$ is given by Eq.(3.26) with $F(z)$ defined in Eq.(C6). For $|z| \ll 1$ this solution for $u(z)$ depends only on $|z|=r$ and is given by Eq.(3.36). 


\section{REFERENCES}

* Present address: Department of Theoretical Physics, University of Lund, Sölvegatan 14A S - 22362 Lund.

${ }^{1}$ J. T.Edwards and D. J. Thouless, J. Phys. C. , 802, (1972); D. J. Thouless, Phys. Rep. 13, 93 (1974).

${ }^{2}$ B. D. Simons and B. L. Altshuler, Phys. Rev. B 48, 5422 (1993); A. Szafer and B. L. Altshuler, Phys. Rev. Lett. 70, 587 (1993); B. D. Simons, P. A. Lee and B. L. Altshuler, Phys. Rev. Lett. 70, 4122 (1993).

${ }^{3}$ E. P. Wigner, Proc. Cambridge Philos. Soc. 47, 790 (1951); F. J. Dyson, J. Math. Phys. 3, 140 (1962).

${ }^{4}$ M. L. Mehta, Random matrices (Academic Press, Boston, 1991).

${ }^{5}$ K. B. Efetov, Adv.Phys. 32, 53 (1983).

${ }^{6}$ A. V. Andreev, O. Agam, B. D. Simons, and B. L. Altshuler, Phys. Rev. Lett. 76, 3947 (1996).

${ }^{7}$ J. Zakrewski and D. Delande, Phys. Rev. E. 47, 1650 (1993).

${ }^{8}$ F. von Oppen, Phys. Rev. E. 51, 2647 (1995);

${ }^{9}$ Y. V. Fyodorov and H.- J. Sommers, Phys. Rev. E. 51, R2719 (1995).

${ }^{10}$ C. M. Canali, C. Basu, W. Stephan and V. E. Kravtsov, Phys. Rev. B 54, 1431 (1996).

${ }^{11}$ M. Titov, D.Braun and Y. V. Fyodorov, J. of Phys. A 30, L339-L345 (1997).

${ }^{12}$ K. Zyczkowski, L. Molinari, and F. M. Izrailev, J. Phys. I France 4, 1469 (1994).

${ }^{13}$ V. E.Kravtsov and I. V.Yurkevich, Phys. Rev. Lett. 78, 3354 (1997).

${ }^{14}$ I. V. Yurkevich and V. E. Kravtsov, Phys. Rev. Lett. 78, 701 (1997).

${ }^{15}$ V. E. Kravtsov and A. D. Mirlin, Pis'ma Zh. Exp. Teor. Fiz. 60, 645 (1994) [JETP Lett. 60, 656 (1994)].

${ }^{16}$ In a ring geometry this twist is equivalent to the effect of a magnetic flux in the problem of persistent current. See M. Buttiker, Y. Imry, and R. Landauer, Phys. Lett. A 96, 365 (1983).

${ }^{17}$ B. L. Altshuler, V. E. Kravtsov and I. V. Lerner in Mesoscopic Phenomena in Solids, ed. B.L.Altshuler et al., (Elsevier, Amsterdam 1991), p.449 and references therein.

${ }^{18}$ V. I. Falko, K. B. Efetov, Phys. Rev. B 52, 17413 (1995).

${ }^{19}$ V. E. Kravtsov, Habilitationsschrift, Heidelberg University 1992 (unpublished).

${ }^{20}$ B. A. Muzykantskii and D. E. Khmelnitskii, Phys. Rev. B 51, 5480 (1995).

${ }^{21}$ I. E. Smolyarenko and B. L. Altshuler, Phys. Rev. B 55, 10451 (1997).

${ }^{22}$ C. Castellani and L. Peliti, J. Phys. A 19, L429 (1986); W. Pook and M. Janssen, Z. Phys. B 82, 295 (1991).

${ }^{23}$ F. Wegner, Z. Phys. B 36, 209 (1980).

${ }^{24}$ Y. V.Fyodorov and A. D. Mirlin, Phys. Rev. B 51, 13403 (1995).

${ }^{25}$ V. E. Kravtsov and M. R.Zirnbauer, Phys. Rev. B 46, 4332 (1992).

${ }^{26}$ This statement is not true ${ }^{25}$ in the case where there is a degeneracy of spectrum at $\phi=0$ (which is lifted for $\phi \neq 0$ ) for different values of T-odd quantities such as spin (Kramers degeneracy) or angular momentum (in perfect systems).

${ }^{27}$ In this paper we have changed sign in the definition of $S T r$ compared to the original Efetov's paper ${ }^{5}$ and Ref. 14. We assume $S \operatorname{Tr} A=\operatorname{Tr} A_{B B}-\operatorname{Tr} A_{F F}$ throughout the paper.

${ }^{28}$ Below the factor $\frac{\pi}{2} g$ is absorbed by the flux $\frac{\pi}{2} g \phi^{2} \rightarrow \phi^{2}$.

${ }^{29}$ J. J. Verbaarschot, H. A. Weidenmuller and M. R. Zirnbauer, Phys. Rep. 129, 367 (1985). 
${ }^{30}$ A. D. Mirlin, Pis'ma Zh. Exp. Teor. Fiz. 62, 583 (1995) [JETP-Lett. 62, 603 (1995)].

${ }^{31}$ A. D. Mirlin, Phys. Rev. B 53, 1186 (1996).

${ }^{32}$ It is always possible by a proper choice of parameters (e.g. the cross-section in quasi-1d case) to implement this limit while keeping the conductance $g$ fixed.

${ }^{33}$ E. Abrahams, P. W. Anderson, D. C Licciardello, and T. V. Ramakrishnan, Phys. Rev. Lett. 42, 673 (1979).

${ }^{34}$ Equation (4.1) is valid in all cases where $g_{d}>>1$. Therefore it is valid in the metallic regime in all dimensions and at the critical point in $d=2+\epsilon$. However in order for the equation to be written in this way one should consider a renormalized $g_{d}=a(d) g$. For the relevant dimensionality we have: $g_{1}=2 \pi g, g_{2}=2 \pi^{2} g$, and $g_{3}=4 \pi^{2} g$, where $g=D /\left(L^{2} \Delta\right)$.

35 J. T. Chalker, Physica A 167, 253 (1990); B. Huckenstein and L. Schweitzer, Phys. Rev. Lett. 72, 713 (1994).

${ }^{36}$ B. L. Altshuler, V. E. Kravtsov, and I. V. Lerner, Zh. Eksp. Teor. Fiz. 91, 2276 (1986) [Sov. Phys. JETP 64, 1352 (1986)].

${ }^{37}$ There was a minor mistake in calculating the Jacobian in Ref. [24]. The correct answer is given by Eq. (B10)

${ }^{38}$ Equation (4.7) is known to be valid for $d=2+\epsilon$ since the work of Refs. [23, 17].

${ }^{39}$ For $\beta=1$ the relation $\mu=2 \beta g_{2}$ follows immediately from Eq.(3.45). For $\beta=2$ it is analogous to the result of Ref.[20] for the problem of current relaxation. Here we are assuming that it remains valid for $\beta=4$ as well.

${ }^{40}$ T. Brandes, B. Huckestein and L. Schweitzer, Ann. Physik 5, 633 (1996); T. Ohtsuki and T. Kawarabayashi, J. Phys. Soc. Jpn. February (1997), and references therein.

${ }^{41}$ R. Klesse and M. Metzler, Phys. Rev. Lett. 79, 721 (1997).

${ }^{42}$ J. T.Chalker and P. D.Coddington, J. Phys. C. 21, 2665 (1988).

${ }^{43}$ P. W.Anderson and P. A.Lee, Prog. Theor. Phys. 69, 212 (1980).

${ }^{44}$ E.Akkermans, J. Math. Phys. 381781 (1997).

${ }^{45}$ D.Braun, E.Hoffstetter, G.Montambaux and A. MacKinnon, Phys. Rev. B 55, 7557 (1997). 


\section{FIGURES}

FIG. 1. Perturbative $1 / g$ correction to the curvature distribution $P(k)$ beyond the RMT result $P_{\mathrm{WD}}(k)$. The expression for $\delta P_{\text {reg }}(k)=P(k)-P_{\mathrm{WD}}(k)$ is given in Eqs. (2.1),(2.2). The dashed line represents the case of global $T$-breaking perturbation (Case I). The solid line represents the case of local $T$-breaking perturbation (Case II).

FIG. 2. Plot of the periodic solution $u(x)$ of the Liouville equation Eq. (3.23), that enters the instanton action of Eq. (3.22), for the quasi-1D case. The function is defined on the ring

$-\frac{1}{2}<x<\frac{1}{2}$. In the interval $-\frac{1}{4}<x<\frac{1}{4}$ the function $u(x)$ is the positive solution of Eq. (3.27) with the choice $b=0$ and $k$ found from the condition $u( \pm 1 / 4)=0$. In the intervals $\pm \frac{1}{2}<x< \pm \frac{1}{4}$ $u(x)$ is constructed by antisymmetric reflection around the points $\pm \frac{1}{4}$.

FIG. 3. Definition domain of the periodic solution $u(z)$ of the Liouville equation, Eq. (3.23), for the $2 d$ case. The domain is a torus $-1 / 2<x, y<1 / 2$, is represented here by the large square. Inside the square $\Omega$ with vertices at $z= \pm 1 / 2, \pm i / 2$ the function $u(z)$ is the real positive solution of Eq. (3.23). In the remaining part of the larger square, $u(z)$ is constructed by anti-symmetric reflection around the sides of the square $\Omega$ and its sign is negative.

FIG. 4. Numerical results for $\delta P(k)=P(k)-P_{\mathrm{WD}}(k)$ for the $3 d$ Anderson model in the metallic regime. The disorder is $w=12 t$ and the system size is $\mathrm{L}=12$. The curvatures are calculated for case I, global vector potential. The smooth curve is fit with the analytical result given in Eqs. (2.1),(2.2). The coefficient $C_{3}$ is taken as a free parameter in the least square fitting.

FIG. 5. The same as in Fig. 4 but for case II, local random magnetic field.

FIG. 6. Numerical results for the difference $\delta P(k)=P(k)-P_{W D}(k)$ for the $3 d$ Anderson model of system size $L=12$ at the Anderson critical point (disorder $W=16.5$ ). The dashed and solid curves are two one-parameter fitting curves provided by by Eq.(2.1) and Eq.(5.4).

FIG. 7. Numerical results for the difference $\delta P(k)=P(k)-P_{W D}(k)$ for the $2 d$ Anderson model of different system sizes $L=12,16,20,24,30$ and site disorder $W=6$.

FIG. 8. Numerical results for the difference $\delta P(k)=P(k)-P_{W D}(k)$ for the $2 d$ Anderson model of system size $L=30$ and site disorder $W=6$. The dashed and solid curves are two one-parameter fitting curves provided by by Eq.(2.1) and Eq.(5.4). 


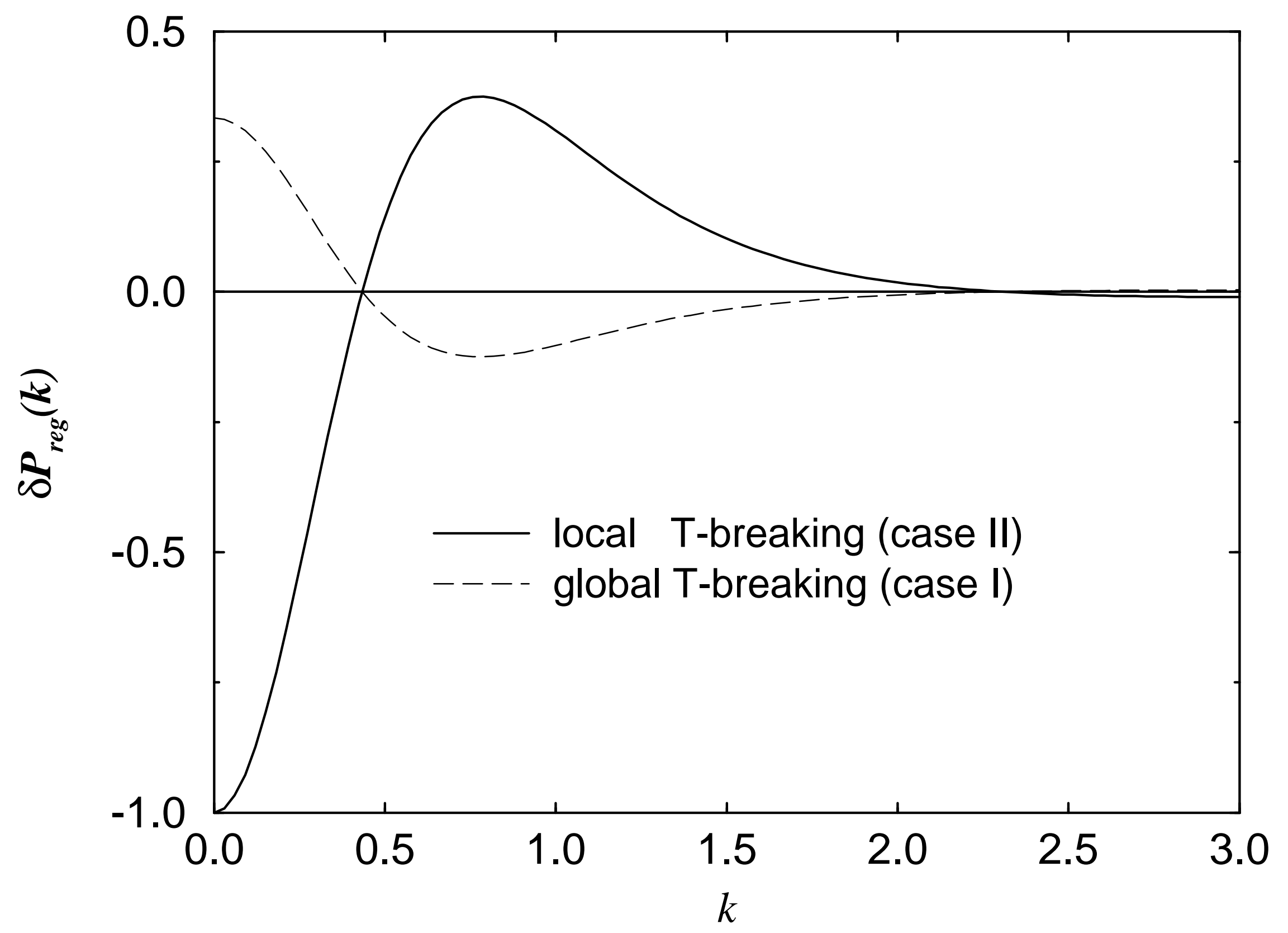




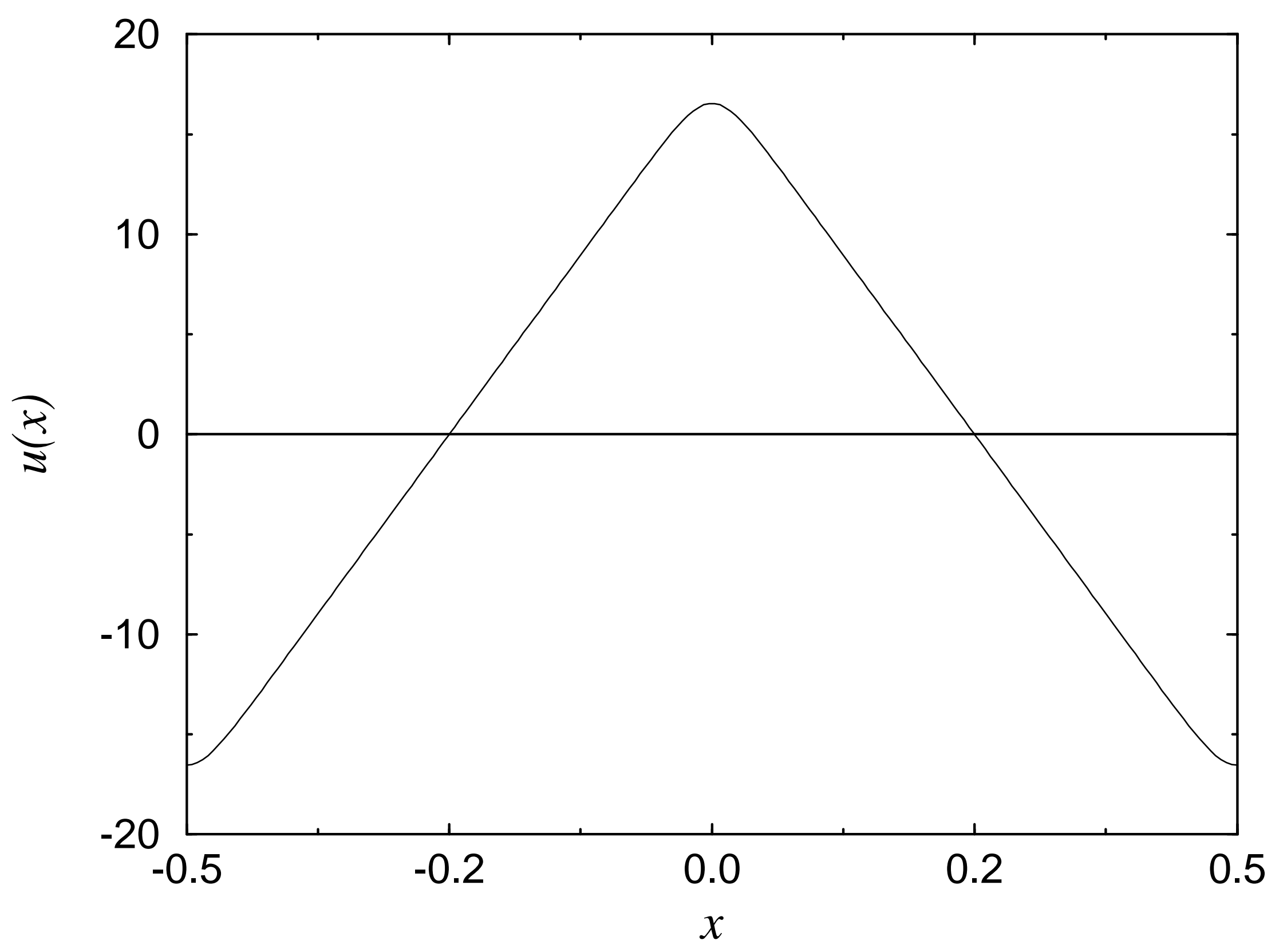




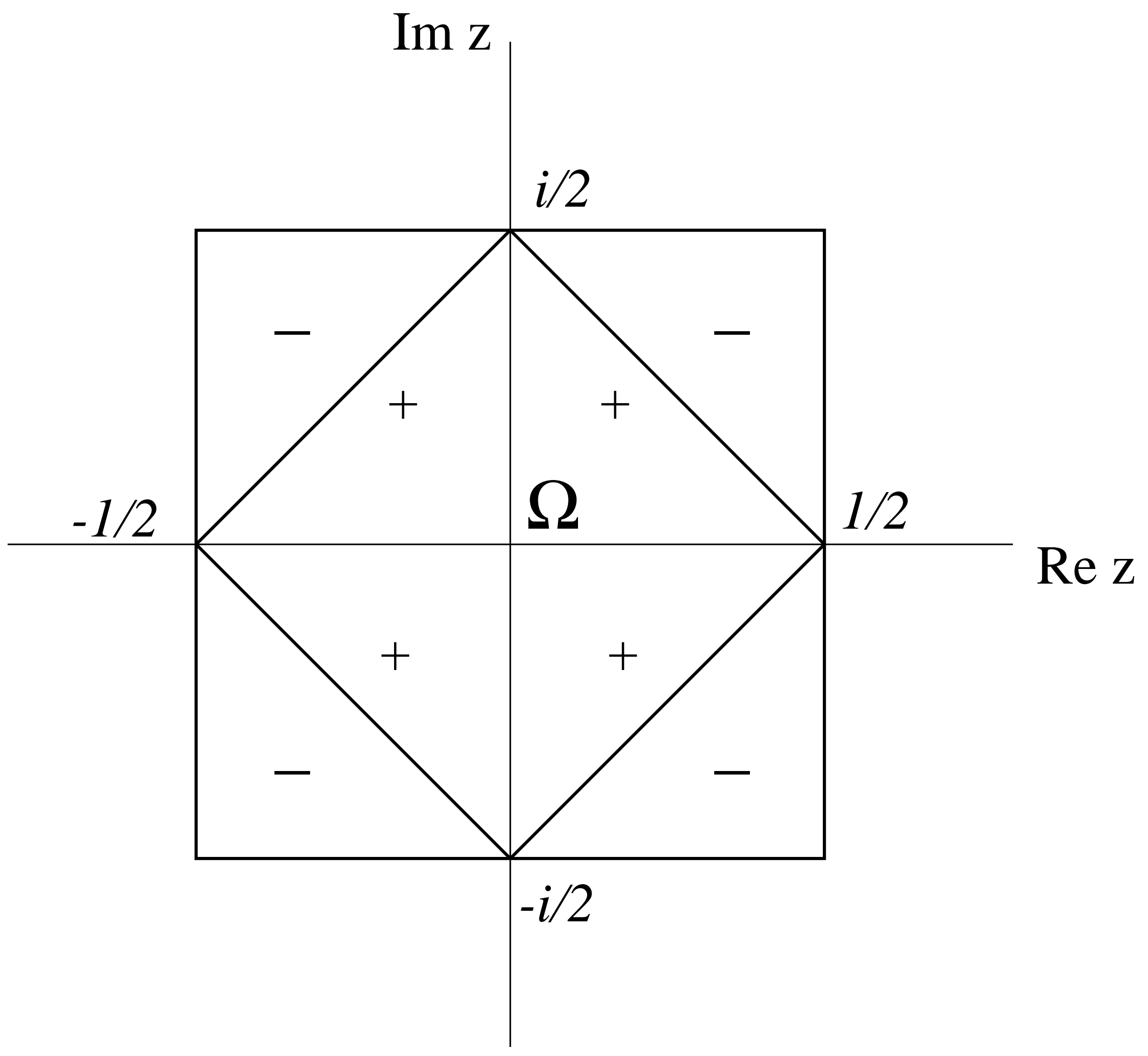




$$
3 d, L=8, W=12
$$

(number of samples $=1500$ )

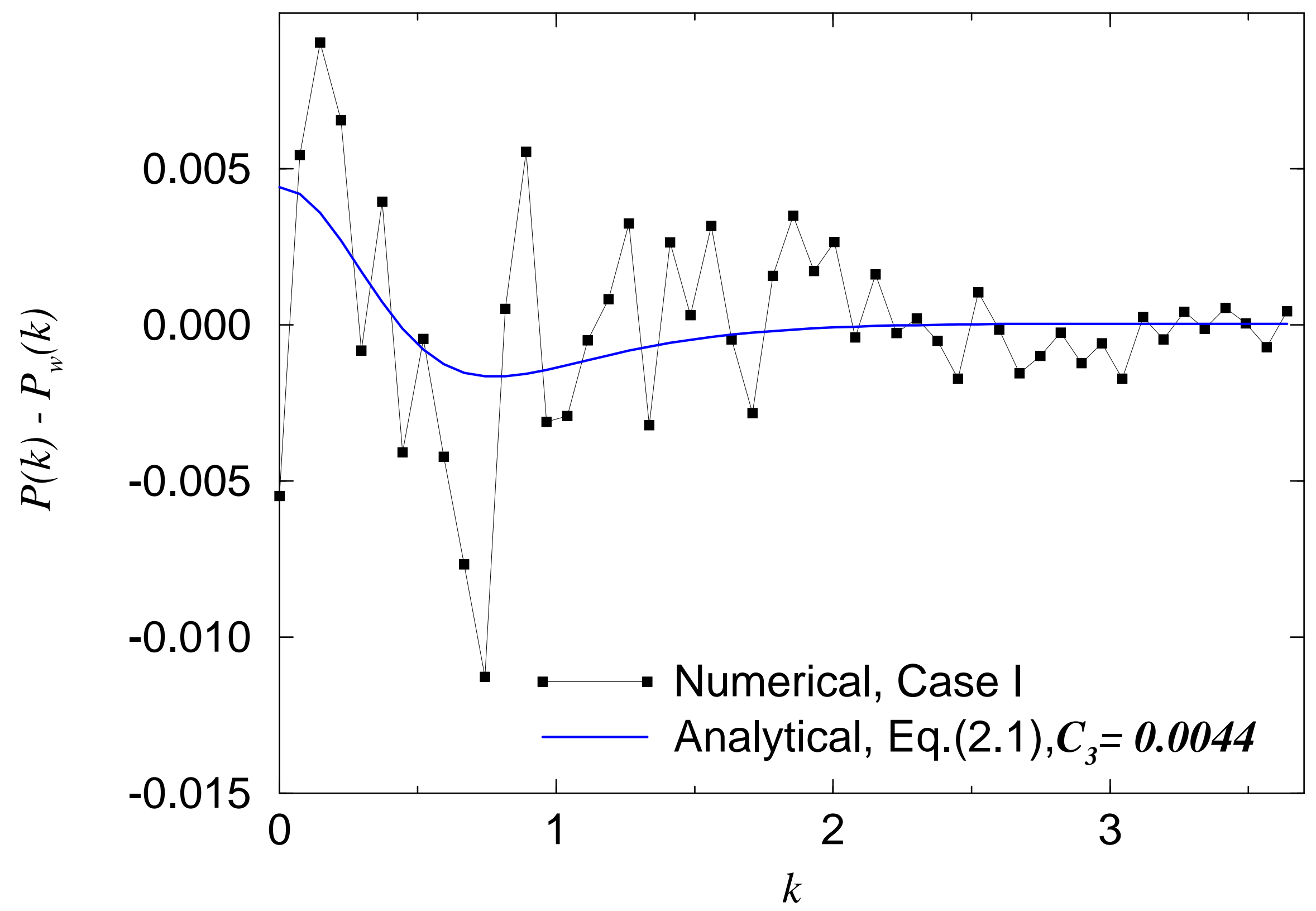




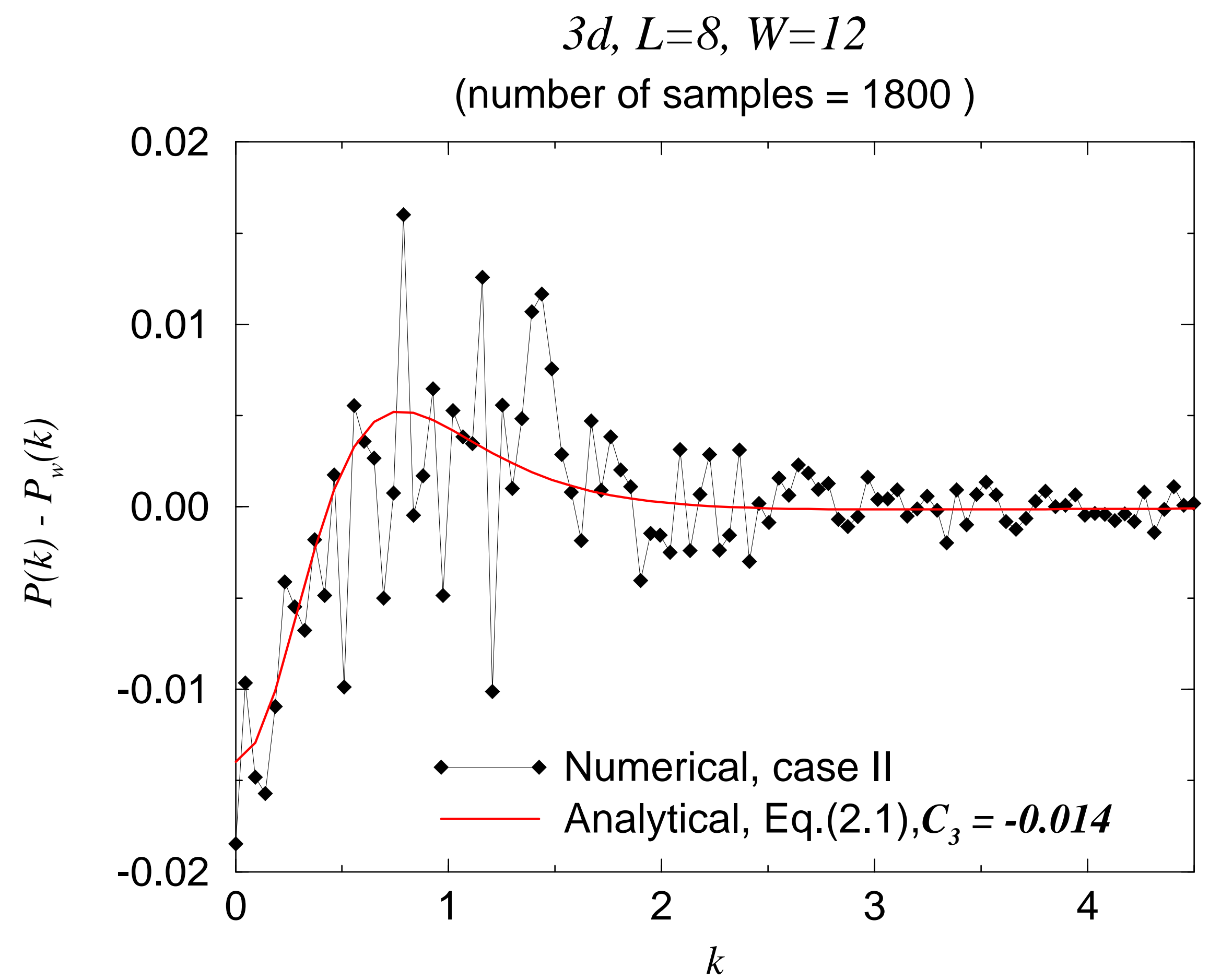




\section{$3 d$, critical point}

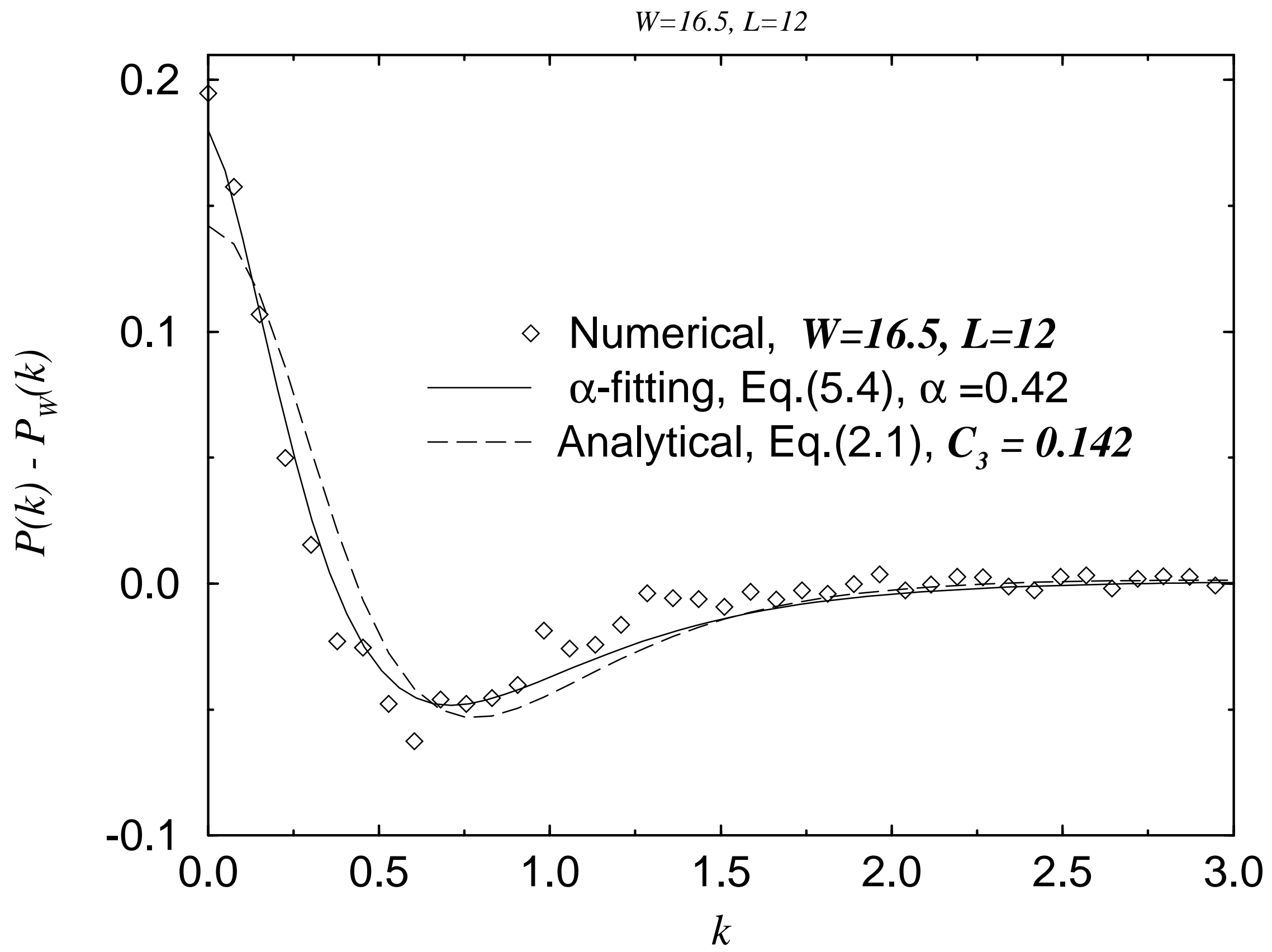


$2 d, W=6$

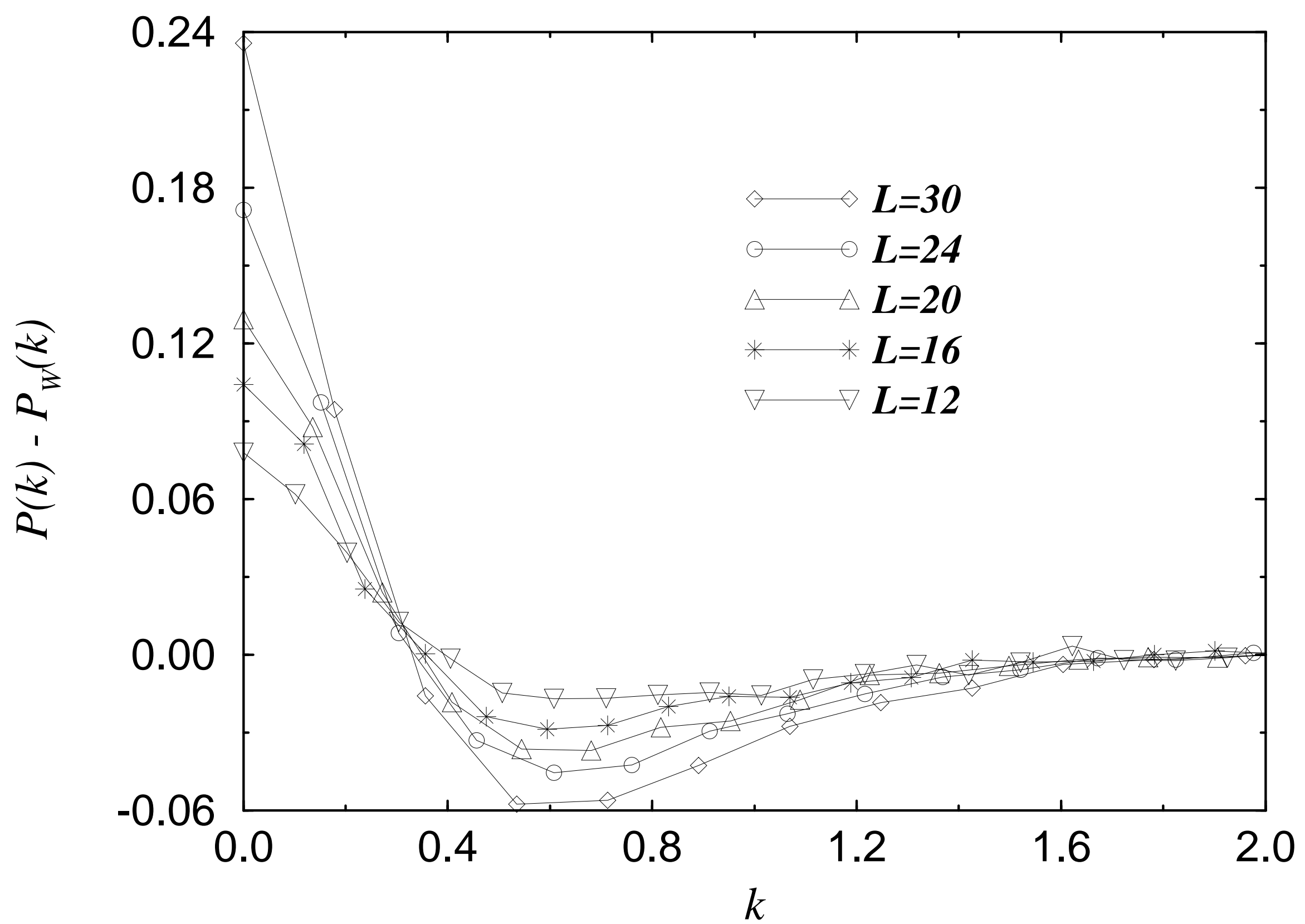




\section{$2 d, W=6$}

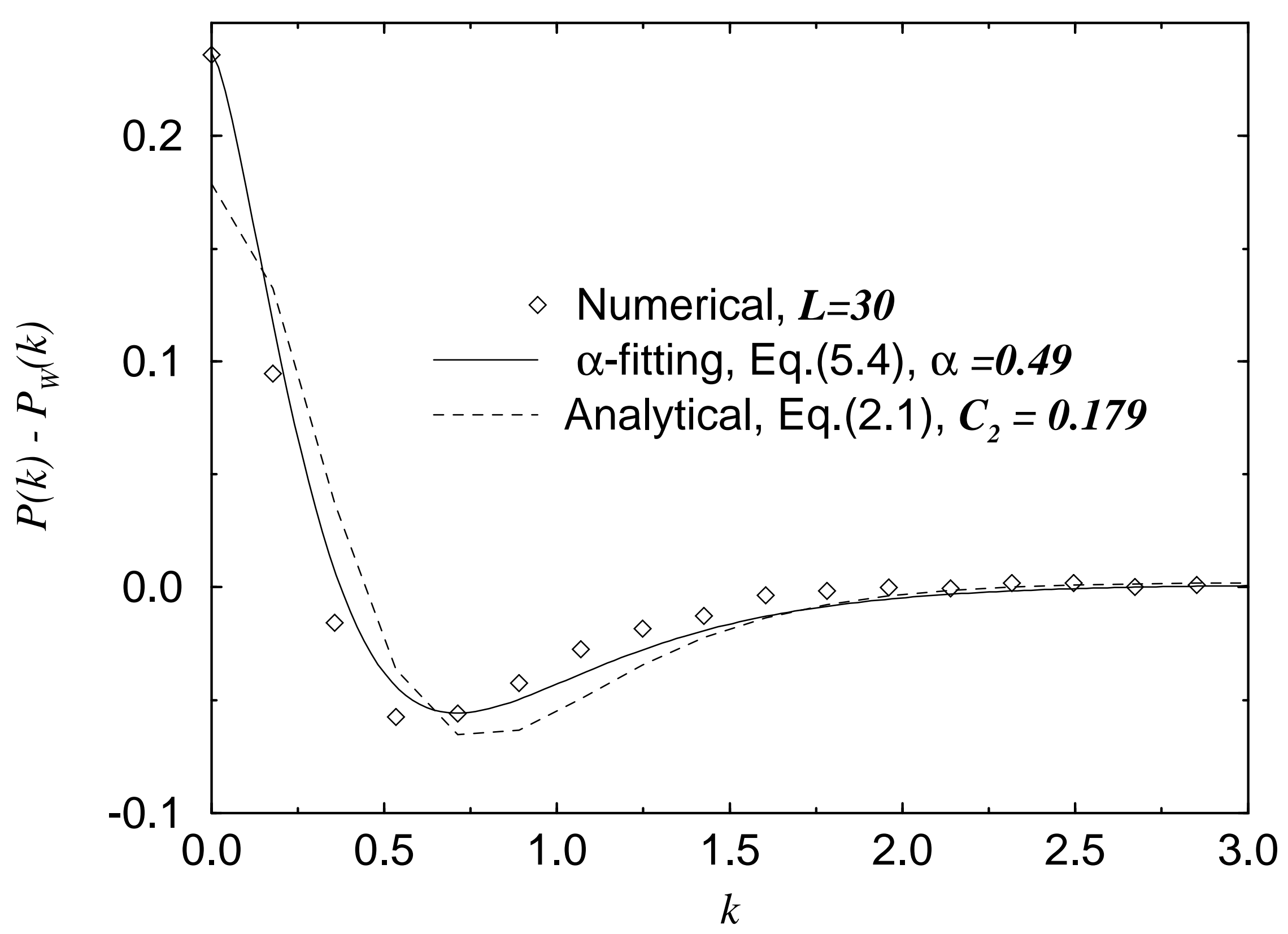

